Alma Mater Studiorum - Università di Bologna DEPARTMENT OF ECONOMICS

\title{
Product Differentiation with Multiple Qualities
}

Francesca Barigozzi

Ching-to Albert Ma

Quaderni - Working Paper DSE $N^{\circ} 1075$

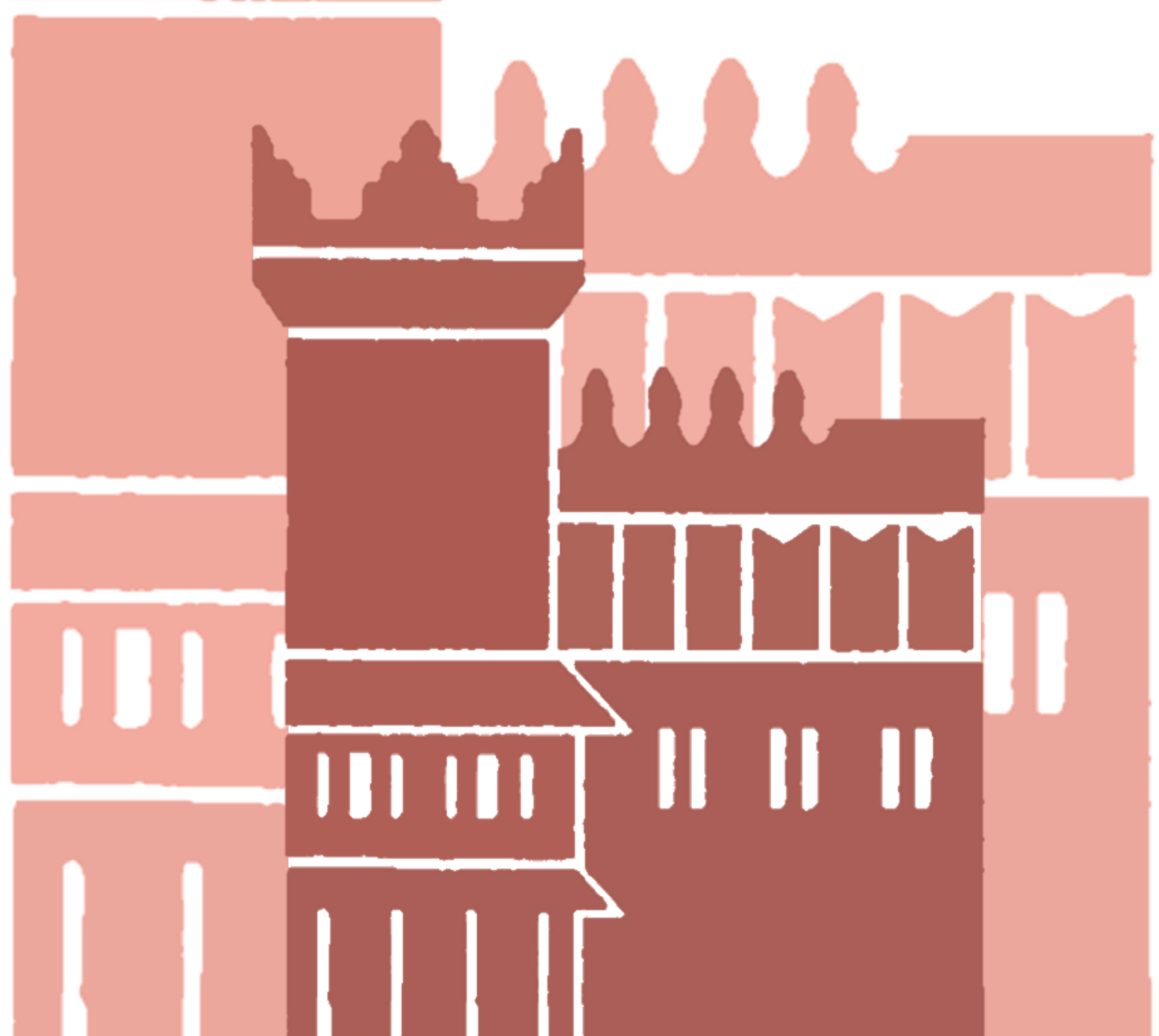




\title{
Product Differentiation with Multiple Qualities
}

\author{
Francesca Barigozzi \\ Department of Economics \\ University of Bologna \\ francesca.barigozzi@unibo.it
}

\author{
Ching-to Albert Ma \\ Department of Economics \\ Boston University \\ ma@bu.edu
}

August 30, 2016

\begin{abstract}
We study subgame-perfect equilibria of the classical quality-price, multistage game of vertical product differentiation. Each firm can choose the levels of an arbitrary number of qualities. Consumers' valuations are drawn from independent and general distributions. The unit cost of production is increasing and convex in qualities. We characterize equilibrium prices, and the equilibrium effects of qualities on the rival's price in the general model. We present necessary and sufficient conditions for equilibrium differentiation in any of the qualities.
\end{abstract}

Keywords: multidimensional product differentiation, quality and price competition

JEL: D43, L13

Acknowledgement: For their comments, we thank Sofronis Clerides, Bard Harstad, Henry Mak, Kjetil Storesletten and seminar participants at Boston University, the University of Oslo, and the 14th International Industrial Organization Conference in Philadelphia. 


\section{Introduction}

Firms using differentiated products to soften intense Bertrand price competition is a basic principle in industrial organization. Following Hotelling (1929), D'Aspremont, Gabszewicz and Thisse (1979) clarify theoretical issues and solve the basic horizontal differentiation model. Gabszewicz and Thisse (1979), and Shaked and Sutton $(1982,1983)$ work out equilibria of the basic vertical differentiation model.

The standard model of horizontal-vertical product differentiation is the following multistage game between two firms: in Stage 1, firms choose product attributes, in Stage 2, firms choose prices, and then consumers pick a firm to purchase from. In the literature, models have seldom gone beyond two possible qualities, have assumed that consumers' quality valuations are uniformly distributed, and have let production or mismatch costs be nonexistent, linear, or quadratic. We make none of these assumptions. In this paper, each of two firms produces goods with an arbitrary number of quality attributes. Consumers' valuations on each quality follow a general distribution. A firm's unit production cost is an increasing and convex function of qualities. In this general environment, we fully characterize subgame-perfect equilibria of the standard differentiation model.

Using the uniform quality-valuation distribution and the separable cost assumptions, researchers have managed to solve for equilibrium prices explicitly as functions of qualities. Equilibrium qualities then can be characterized. What has emerged in the literature are a few classes of equilibria with largest or smallest differences in equilibrium qualities (see the literature review in the following subsection). Indeed, in equilibrium firms may successfully differentiate their products in some qualities, but may fail to do so in others. Obviously, tractability versus generality is the challenge that has been posed by the literature. How robust are maximal or minimal differentiation results? To what extent are they driven by these assumptions?

In this paper we solve the tractability-generality dilemma. For the quality-price, multistage game, we completely characterize subgame-perfect equilibria. First, we find out how qualities change equilibrium prices - without solving for the equilibrium prices explicitly in terms of qualities. In other words, we do not need the usual assumptions in order to compute equilibrium prices explicitly. Second, we identify two separate effects for the characterization of equilibrium qualities. The first is what we call the price-reaction 
effect, which is how a firm's quality in Stage 1 affects the rival firm's price in Stage 2. The second is what we call the Spence effect (because it is originally exposited in Spence (1975); see footnote 7). For maximum profit, a firm chooses a quality which is efficient for the consumer who is just indifferent between buying from the firm and its rival.

Consumers must buy from one of the two firms, so firms share the same set of indifferent consumers. The Spence effect says that each firm should choose those qualities that are efficient for the equilibrium set of indifferent consumers. The Spence effect alone is a motivation for minimal product differentiation: for each quality, firms should choose the same level. But there is a second effect at work.

In the standard multistage game, prices are strategic complements. In Stage 1, each firm would like to use its qualities to induce the rival to choose a higher price in Stage 2. The rival firm's strategic consideration, however, is exactly the same. Hence, the two firms engage in a race. For each quality, the relative strength of the price-reaction effect determines which firm will, in equilibrium, choose a higher quality than its rival. For general distributions of quality valuations and a general cost function, it is entirely possible for the relative strength to vary across different qualities. The price-reaction effect implies that firms may differentiate their products in many ways. Largest or smallest differences in equilibrium qualities are not robust. Instead, we provide a full characterization of how equilibrium qualities may differ between firms.

Our results say that firms choosing the same level of a quality holds under very restrictive conditions: cost is separable across quality attributes together with consumers' quality valuations being uniformly distributed (see Corollary 3 below). However, these have been the exact assumptions used in the literature. One casually observes that quality attributes across products are never exactly the same. Thus, firms that produce "highend" products will still differentiate - if only in small details of their product qualities. For example, $B M W$ and Lexus are companies that differentiate even in the high qualities of their cars. All BMW and Lexus cars are high-quality automobiles, but the common consensus is that $B M W$ has a higher "performance" quality than Lexus, but the opposite is true when it comes to the "comfort" quality. However, any car by $B M W$ or Lexus will be a better performer and more comfortable than any car by Yugo. In fact, in the automobile (and most other) markets, it is impossible to find products that have identical quality attributes. 
These observations are consistent with the general tenet of product differentiation, but inconsistent with assumptions of uniform quality-valuation distribution and separable cost functions.

What is behind our solution to the tractability-generality dilemma? Our key innovation is to show that solving for the equilibrium prices as functions of qualities is equivalent to solving a single integral equation. The solution to the integral equation yields the equilibrium set of indifferent consumers, and hence the firms' demands. We obtain this solution as an implicit function of the model primitives. Then a firm's equilibrium price can be expressed explicitly in terms of qualities, through the solution of the integral equation. In other words, we dispense with the need for explicit computation of equilibrium prices, which would require explicit specification of the model primitives, a common research strategy in the literature. Judicious analysis of mutual quality best responses, given qualities' impact on equilibrium prices, yields the fundamental pricereaction and Spence effects. For many examples, the integral equation does admit an explicit solution (that will be demonstrated later).

We use a vertical differentiation model, but Cremer and Thisse (1991) show that for the usual model specification, the Hotelling, horizontal differentiation model is a special case of the vertical differentiation model. The intuition is simply that firms' demand functions in a Hotelling model can be directly translated to the demand functions in a vertical, quality model. Cremer and Thisse (1991) state the result for a single location or quality dimension, but their result extends straightforwardly to an arbitrary number of such dimensions. (A model with a combination of horizontal and vertical dimensions can also be translated to a model with only vertical dimensions.) Hence our results in this paper apply to horizontal-differentiation models. In particular, our method of solving for equilibrium prices is valid for Hotelling models. ${ }^{1}$

We continue with a subsection on the literature. In Section 2, we define consumers' preferences and firms' technology. Then we set up the quality-price, multistage game. Section 3 is divided into four subsections. In Subsection 3.1, we characterize subgame-perfect equilibrium prices. Lemma 1 presents the solution of the integral equation, the key step in expressing equilibrium prices as functions of qualities. In Subsection 3.2,

\footnotetext{
${ }^{1}$ Differences between horizontal and vertial models may also be due to specification of the strategy sets. Here, we allow qualities to take any positive values; in location models, firms' positions may not vary as much. See also the discussions following Corollary 3 .
} 
we characterize how prices change with qualities. In Subsection 3.3, we characterize equilibrium qualities, and establish the price-reaction and Spence effects. Subsection 3.4 presents a number of implications. We specialize our model by adopting common assumptions (uniform quality-valuation distribution and separable cost function), and draw connections between earlier results and ours. A number of examples are studied in Section 4. These examples illustrate how our general results can be used. The last section contains some concluding remarks. Proofs of results are in Appendix A, and some steps for computation for Subsection 4.3 are in Appendix B.

\subsection{Literature review}

The modern literature on product differentiation and competition begins with D'Aspremont, Gabszewicz and Thisse (1979), Gabszewicz and Thisse (1979) and Shaked and Sutton $(1982,1983)$. In the past few decades, the principle of product differentiation relaxing price competition has been stated in texts of industrial organization at all levels: Tirole (1988), Anderson, De Palma, and Thisse (1992), and Belleflamme and Peitz (2010) for graduate level, as well as Cabral (2000), Carlton and Perloff (2005), and Pepall, Richards, and Norman (2014). Many researchers use the basic horizontal and vertical differentiation models as their investigation workhorse.

The research here focuses on equilibrium differentiation. In both horizontal and vertical models, a common theme has been to solve for subgame-perfect equilibria in the quality-price, multistage game in various environments. ${ }^{2}$ First, earlier papers have looked at single or multiple horizontal and vertical dimensions of consumer preferences. Second, most papers have adopted the assumption that these preferences are uniformly distributed. Third, most papers in the horizontal model have used a quadratic consumer mismatch disutility function, whereas those in the vertical model have assumed that the unit production cost is either independent of, or linear in quality.

\section{Single dimension of preferences}

Anderson, Goeree and Ramer (1997) study equilibrium existence and characterization in a single-dimension

\footnotetext{
${ }^{2}$ As we have already mentioned, Cremer and Thisse (1991) (following on a suggestion by Champsaur and Rochet (1989)) show that horizontal-location models are special cases of vertical models.
} 
horizontal model. They use a general consumer preference distribution but quadratic mismatch disutility. Our multidimensional vertical model can be recast into the single-dimension model in Anderson, Goeree and Ramer, and we will demonstrate that in Subsection 4.2. A few other papers have adopted nonuniform distributions on consumer locations. Neven (1986) shows that firms tend to locate inside the market when consumers' densities are higher near the center. Tabuchi and Thisse (1995) assume a triangular distribution and find that there is no symmetric location equilibria but that asymmetric location equilibria exist. Yurko (2010) uses a vertical model for studying entry decisions, but her results are based on numerical simulations. Benassi, Chirco, and Colombo (2006) allow consumers the nonpurchase option. They relate various trapezoidal valuation distributions to degrees of equilibrium quality differentiation. Finally, Loertscher and Muehlheusser (2011) consider entry in location games without price competition. They study equilibria under the uniform and some nonuniform consumer-location distributions.

\section{Multiple dimensions of vertical preferences}

A few papers have studied vertical models with two dimensions. These are Vandenbosch and Weinberg (1995), Lauga and Ofek (2011), and Garella and Lambertini (2014). All three papers use the uniform valuation distribution. In Subsection 3.4, we will present the relationship between our results here to those

in these papers. Here, we note that these papers have assumed zero production cost, or unit cost that is linear or discontinuous in quality. By contrast we use a strictly convex quality cost function.

\section{Multiple dimensions of horizontal preferences}

For horizontal models with multiple dimensions, the key paper is Irmen and Thisse (1998), who set up an $N$ dimensional model to derive what they call "Max-Min-...Min" equilibria. We will relate our results to those in Irmen and Thisse in Subsection 3.4, right after Corollary 4. Tabuchi (1994) and Vendorp and Majeed (1995) are special cases of Irmen and Thisse (1998) at $N=2$. Ansari, Economides, and Steckel (1998) study two and three dimensional Hotelling models, and derive similar results as in Irmen and Thisse (1998). All assume that consumers' locations are uniformly distributed, and that the mismatch disutility is Euclidean and therefore separable. We are unaware of any paper in the multidimensional horizontal literature that adopts general consumer preferences distributions, or general, nonseparable mismatch disutility. 
Finally, Degryse and Irmen (2001) use a model with both horizontal and vertical differentiation. For the horizontal dimension, consumer locations are uniformly distributed. For the vertical dimension, consumers have the same valuation (as in the model in Garella and Lambertini (2014)). However, the mismatch disutility depends also on quality, which corresponds to the case of a nonseparable mismatch disutility or quality cost function. This can be thought of as a special case of the model here; see Subsection 4.2.

\section{The Model}

We begin with consumers and their preferences. Then we present two identical firms. Finally, we define demands, profits, and the extensive form of quality-price competition.

\subsection{Consumers and preferences for qualities}

There is a set of consumers, with total mass normalized at 1. Each consumer would like to buy one unit of a good, which has $N \geq 2$ quality attributes. A good is defined by a vector of qualities $\left(q_{1}, q_{2} \ldots, q_{N}\right) \in \Re_{+}^{N}$, where $q_{i}$ is the level of the $i^{t h}$ quality, $i=1,2, \ldots, N$. Sometimes, we use the term quality $q_{i}$ to mean the level of quality attribute $i$.

A consumer's preferences on goods are described by his quality valuations, represented by the vector $\left(v_{1}, \ldots, v_{i}, \ldots, v_{N}\right) \in \prod_{i=1}^{N}\left[\underline{v}_{i}, \bar{v}_{i}\right] \subset \Re_{++}^{N}$. The valuation on quality $q_{i}$ is $v_{i}$, which varies in a bounded, and strictly positive interval. If a consumer with valuation vector $\left(v_{1}, \ldots, v_{i}, \ldots, v_{N}\right) \equiv v$ buys a good with qualities $\left(q_{1}, q_{2} \ldots, q_{N}\right) \equiv q$ at price $p$, his utility is $v_{1} q_{1}+v_{2} q_{2}+\ldots+v_{N} q_{N}-p$. (We may sometimes call this consumer $\left(v_{1}, \ldots, v_{N}\right)$ or simply consumer $v$.) The quasi-linear utility function is commonly adopted in the literature (see such standard texts as Tirole (1988) and Belleflamme and Peitz (2010))

Consumers' heterogeneous preferences on qualities are modeled by letting the valuation vector be random. We use the standard independence distribution assumption: the valuation $v_{i}$ follows the distribution function $F_{i}$ with the corresponding density $f_{i}, i=1, \ldots, N$, and these distributions are all independent. Each density is assumed to be differentiable (almost everywhere) and logconcave. The logconcavity of $f_{i}$ implies that the 
joint density of $\left(v_{1}, \ldots, v_{i}, \ldots, v_{N}\right) \equiv v$ is logconcave, ${ }^{3}$ and it guarantees that profit functions, to be defined below, are quasi-concave (see Proposition 4 in Caplin and Nalebuff 1991, p39).

\subsection{Firms and extensive form}

There are two firms and they have access to the same technology. If a firm produces a good at quality vector $\left(q_{1}, q_{2} \ldots, q_{N}\right) \equiv q$, the per-unit production cost is $C(q)$. There is no fixed cost, so if a firm produces $D$ units of the good at quality $q$, its total cost is $D$ multiplied by $C(q)$. We assume that the per-unit quality cost function $C: \mathbb{R}_{+}^{N} \rightarrow \mathbb{R}_{+}$is strictly increasing and strictly convex. We also assume that $C$ is continuous and differentiable, and satisfies the usual Inada conditions: $\lim _{q \rightarrow 0} C(q)=\lim _{q \rightarrow 0} \mathrm{~d} C(q)=0$ (where 0 stands for either the number zero or an $N$-vector of all zeros), and that $\lim _{q \rightarrow \infty} C(q)=\lim _{q \rightarrow 0} \mathrm{~d} C(q)=\infty$ (again $\infty$ stands for infinity or an $N$-vector of infinities). ${ }^{4}$ If a firm sells $D$ units of the good with quality vector $q$ at price $p$, its profit is $D \cdot[p-C(q)]$.

The two firms are called Firm $A$ and Firm $B$. We use the notation $q$ for Firm $A$ 's vector of qualities $\left(q_{1}, q_{2}, \ldots \ldots q_{N}\right) \equiv q$. We use the notation $r$ for Firm $B$ 's vector of qualities $\left(r_{1}, r_{2}, \ldots \ldots . r_{N}\right) \equiv r$. Hence, when we say quality $q_{i}$, it indicates the level of Firm $A$ 's quality attribute $i$, whereas when we say quality $r_{i}$, it indicates the level of Firm $B$ 's quality attribute $i$. Let Firm $A$ 's price be $p_{A}$, and Firm $B$ 's price be $p_{B}$. We use the notation $p$ for the price vector $\left(p_{A}, p_{B}\right)$.

Given the two firm's quality choices, consumer $v=\left(v_{1}, . ., v_{N}\right)$ obtains utilities $v_{1} q_{1}+v_{2} q_{2}+\ldots+v_{N} q_{N}-p_{A}$ or $v_{1} r_{1}+v_{2} r_{2}+\ldots+v_{N} r_{N}-p_{B}$ from Firm $A$ or Firm $B$, respectively. Consumer $v$ purchases from Firm $A$ if and only if $v \cdot q-p_{A}>v \cdot r-p_{B}$. If the consumer is indifferent because $v \cdot q-p_{A}=v \cdot r-p_{B}$, he picks a firm to buy from with probability 0.5 . For given quality vectors and prices, the demands for Firm $A$ and Firm $B$ are, respectively,

$$
\int \cdot \iint_{v \cdot q-p_{A} \geq v \cdot r-p_{B}} \mathrm{~d} F_{1} \mathrm{~d} F_{2} \cdot \mathrm{d} F_{N} \quad \text { and } \quad \int . \iint_{v \cdot q-p_{A} \leq v \cdot r-p_{B}} \mathrm{~d} F_{1} \mathrm{~d} F_{2} \cdot \mathrm{d} F_{N} .
$$

\footnotetext{
${ }^{3}$ Because of independence, the joint density of $\left(v_{1}, \ldots, v_{i}, \ldots, v_{N}\right)$ is $\prod_{i=1}^{N} f_{i}$. Hence, $\ln \prod f_{i}=\sum \ln f_{i}$. Because $\ln f_{i}$ is concave, so is $\sum \ln f_{i}$.

${ }^{4}$ We have used a general cost function, whereas papers in the extant literature adopt various more restrictive assumptions. Popular simplifications include $C$ being separable in qualities, $C$ being linear, or even identically zero. More discussions are in Subsection 3.4.
} 
The two firms' profits are

$$
\begin{gathered}
\left\{\int_{v \cdot q-p_{A} \geq v \cdot r-p_{B}} \cdot \iint_{v} \mathrm{~d} F_{1} \mathrm{~d} F_{2} \cdot \mathrm{d} F_{N}\right\}\left[p_{A}-C(q)\right] \equiv \pi_{A}\left(p_{A}, p_{B} ; q, r\right) \\
\left\{\int_{v \cdot q-p_{A} \leq v \cdot r-p_{B}} \ldots \iint_{1} \mathrm{~d} F_{1} \mathrm{~d} F_{2} \cdot \mathrm{d} F_{N}\right\}\left[p_{B}-C(r)\right] \equiv \pi_{B}\left(p_{A}, p_{B} ; q, r\right) .
\end{gathered}
$$

In case $q=r$, and $p_{A}=p_{B}$, each firm sells to one half of the mass of consumers.

We study subgame-perfect equilibria of the standard multistage game of quality-price competition:

Stage 0: Consumers valuations are drawn from respective distributions.

Stage 1: Firm $A$ and Firm $B$ simultaneously choose their product qualities $q$ and $r$, respectively.

Stage 2: Firm $A$ and Firm $B$ simultaneously choose their product prices. Then each consumer picks a firm to buy from.

\section{Equilibrium product differentiation}

We begin with the subgame in Stage 2, defined by a pair of quality vectors chosen by firms in Stage 1 . Let Firm $A$ 's qualities be $\left(q_{1}, q_{2}, \ldots, q_{N}\right) \equiv q$, and let Firm $B$ 's qualities be $\left(r_{1}, r_{2}, \ldots r_{N}\right) \equiv r$. If Firm $A$ 's and Firm $B$ 's prices are $p_{A}$ and $p_{B}$, respectively, consumer $v=\left(v_{1}, \ldots, v_{N}\right)$ now buys from Firm $A$ if $v \cdot q-p_{A}>v \cdot r-p_{B}$. The set of consumers who are indifferent between buying from Firm $A$ and Firm $B$ is given by the equation $v_{1} q_{1}+\ldots+v_{N} q_{N}-p_{A}=v_{1} r_{1}+\ldots+v_{N} q_{N}-p_{B}$. For $q_{1} \neq r_{1}$ we solve for $v_{1}$ in this equation to define the following function:

$$
\widetilde{v}_{1}\left(v_{-1} ; p, q, r\right) \equiv \frac{p_{B}-p_{A}}{r_{1}-q_{1}}-\sum_{k=2}^{N} v_{k} \frac{r_{k}-q_{k}}{r_{1}-q_{1}}
$$

where $v_{-1}=\left(v_{2}, \ldots, v_{N}\right)$ is the vector of valuations of the second to the last quality attributes. The vector

$\left(\widetilde{v}_{1}\left(v_{-1} ; p, q, r\right), v_{2}, \ldots, v_{N}\right) \equiv\left(\widetilde{v}_{1}\left(v_{-1} ; p, q, r\right), v_{-1}\right)$ describes all consumers who are indifferent between buying from the two firms.

The function $\widetilde{v}_{1}$ in $(3)$ is linear in the valuations, and this is an important property from the quasi-linear consumer utility function. The function is illustrated in Figure 1 for the case of two qualities $(N=2)$. 


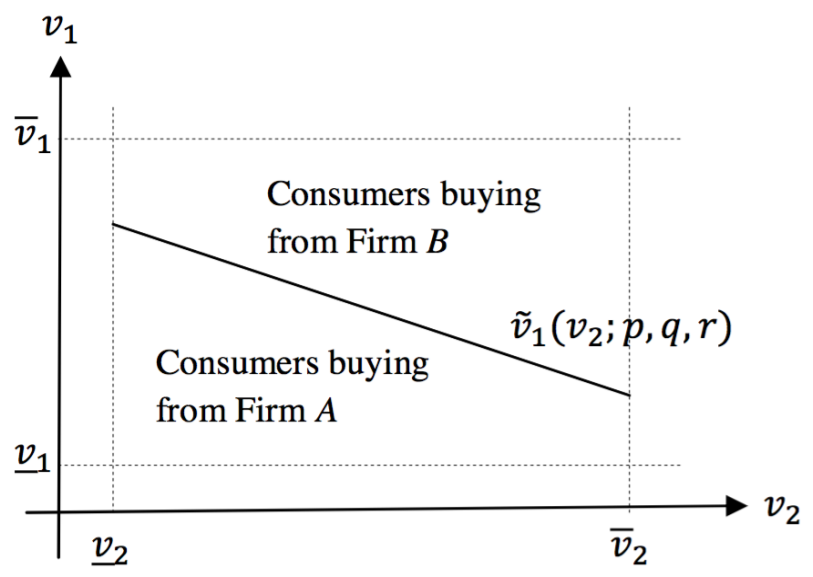

Figure 1: Consumers' choices given prices and qualities

There, we have the valuation $v_{1}$ on the vertical axis, and the valuation $v_{2}$ on the horizontal axis. For this illustration, we have set $\left(q_{1}, q_{2}\right)<\left(r_{1}, r_{2}\right)$ and $p_{A}<p_{B}$. The function $\widetilde{v}_{1}$ is the negatively sloped straight line with the formula $\widetilde{v}_{1}\left(v_{2} ; p, q, r\right)=\frac{p_{B}-p_{A}}{r_{1}-q_{1}}-v_{2} \frac{r_{2}-q_{2}}{r_{1}-q_{1}}$. Prices affect only the intercept, whereas qualities affect both the intercept and the slope.

We further consider the case of $q_{1}<r_{1}$. Consumer $\left(v_{1}^{\prime}, v_{2}, \ldots v_{N}\right)$ buys from Firm $B$ if and only if $v_{1}^{\prime}>\widetilde{v}_{1}\left(v_{-1} ; p, q, r\right)$. In other words, if quality $r_{1}$ is higher than quality $q_{1}$, Firm $B$ 's product is more attractive to a consumer with a higher valuation $v_{1}$. In Figure 1, the set of consumers who buy from Firm $B$ consists of those above $\widetilde{v}_{1}$. Hence, we reformulate the firms' demands as:

Firm $A$

$$
\begin{aligned}
& \int_{\underline{v}_{N}}^{\bar{v}_{N}} \ldots \int_{\underline{v}_{2}}^{\bar{v}_{2}} \int_{\underline{v}_{1}}^{\bar{v}_{1}} \Pi_{i=1}^{N} \mathrm{~d} F_{i}\left(v_{i}\right) \\
& =\int_{\underline{v}_{N}}^{\widetilde{v}_{N}} \ldots \int_{\underline{v}_{2}}^{\bar{v}_{2}} F_{1}\left(\widetilde{v}_{1}\left(v_{-1} ; p, q, r\right)\right) \Pi_{k=2}^{N} \mathrm{~d} F_{k}\left(v_{k}\right)
\end{aligned}
$$

Firm $B$

$$
\begin{aligned}
& \int_{\underline{v}_{N}}^{\bar{v}_{N}} \ldots \int_{\underline{v}_{2}}^{\bar{v}_{2}} \int_{\widetilde{v}_{1}}^{\bar{v}_{2}} \prod_{i=1}^{\bar{v}_{1}} \mathrm{~d} F_{i}\left(v_{i}\right) \\
& =\int_{\underline{v}_{N}}^{\bar{v}_{N}} \ldots \int_{\underline{v}_{2}}^{\bar{v}_{2}}\left[1-F_{1}\left(\widetilde{v}_{1}\left(v_{-1} ; p, q, r\right)\right)\right] \Pi_{k=2}^{N} \mathrm{~d} F_{k}\left(v_{k}\right) .
\end{aligned}
$$

For some values of $q, r$, and prices $p_{A}$ and $p_{B}$, as $v_{-1}$ varies over its ranges, the value of the formula in (3) may be outside the support $\left[\underline{v}_{1}, \bar{v}_{1}\right]$. We can formally include these possibilities by extending the valuation support over the entire real line, but set $f_{1}(x)=0$ whenever $x$ lies outside the support $\left[\underline{v}_{1}, \bar{v}_{1}\right]$. For easier exposition, we will stick with the current notation. 
We use the following shorthand to simplify the notation:

$$
\int_{v_{-1}} \text { stands for } \int_{\underline{v}_{N}}^{\bar{v}_{N}} \ldots \int_{\underline{v}_{2}}^{\bar{v}_{2}} \text { and } \mathrm{d} F_{-1} \quad \text { stands for } \Pi_{k=2}^{N} \mathrm{~d} F_{k}\left(v_{k}\right)
$$

Profits of Firms $A$ and $B$ are, respectively:

$$
\begin{aligned}
& \pi_{A}\left(p_{A}, p_{B} ; q, r\right)=\int_{v_{-1}} F_{1}\left(\widetilde{v}_{1}\left(v_{-1} ; p, q, r\right)\right) \mathrm{d} F_{-1} \times\left[p_{A}-C(q)\right] \\
& \pi_{B}\left(p_{A}, p_{B} ; q, r\right)=\int_{v_{-1}}\left[1-F_{1}\left(\widetilde{v}_{1}\left(v_{-1} ; p, q, r\right)\right)\right] \mathrm{d} F_{-1} \times\left[p_{B}-C(r)\right] .
\end{aligned}
$$

\subsection{Subgame-perfect equilibrium prices}

A subgame in Stage 2 is defined by the firms' qualities. Let Firm A's quality vector be $q$ and Firm $B$ 's quality vector be $r$, so we identify a subgame in Stage 2 by quality vectors $(q, r)$. It is clear that if $q=r$, the equilibrium in Stage 2 is the standard Bertrand equilibrium so each firm will charge its unit production cost: $p_{A}=p_{B}=C(q)=C(r)$.

We now turn to subgames in which $q \neq r$. By a permutation of quality indexes and interchanging the firms' indexes if necessary, we let $q_{1}<r_{1}$. A price equilibrium in subgame $(q, r)$ is a pair of prices $\left(p_{A}^{*}, p_{B}^{*}\right)$ that are best responses: $p_{A}^{*}=\operatorname{argmax}_{p_{A}} \pi_{A}\left(p_{A}, p_{B}^{*} ; q, r\right)$ and $p_{B}^{*}=\operatorname{argmax}_{p_{B}} \pi_{B}\left(p_{A}^{*}, p_{B} ; q, r\right)$, where the profit functions are defined by (4) and (5). The existence of a price equilibrium follows from Caplin and Nalebuff (1991). Furthermore, because of the logconcavity assumption on the densities, a firm's profit function is quasi-concave in its own price.

As we will show, the characterization of equilibrium prices boils down to the properties of the solution of an integral equation. We begin with differentiating the profit functions with respect to prices:

$$
\begin{aligned}
\frac{\partial \pi_{A}}{\partial p_{A}} & =\int_{v_{-1}} F_{1}\left(\widetilde{v}_{1}\left(v_{-1} ; p, q, r\right)\right) \mathrm{d} F_{-1}-\int_{v_{-1}} f_{1}\left(\widetilde{v}_{1}\left(v_{-1} ; p, q, r\right)\right) \mathrm{d} F_{-1}\left[\frac{p_{A}-C(q)}{r_{1}-q_{1}}\right] \\
\frac{\partial \pi_{B}}{\partial p_{B}} & =\int_{v_{-1}}\left[1-F_{1}\left(\widetilde{v}_{1}\left(v_{-1} ; p, q, r\right)\right)\right] \mathrm{d} F_{-1}-\int_{v_{-1}} f_{1}\left(\widetilde{v}_{1}\left(v_{-1} ; p, q, r\right)\right) \mathrm{d} F_{-1}\left[\frac{p_{B}-C(r)}{r_{1}-q_{1}}\right],
\end{aligned}
$$

where we have used the derivatives of $\widetilde{v}_{1}\left(v_{-1} ; p, q, r\right)$ in (3) with respect to $p_{A}$ and $p_{B}$. 
The equilibrium prices $\left(p_{A}^{*}, p_{B}^{*}\right) \equiv p^{*}$ satisfy the first-order conditions:

$$
\begin{aligned}
p_{A}^{*}-C(q)= & \frac{\int_{v_{-1}} F_{1}\left(\widetilde{v}_{1}\left(v_{-1} ; p^{*}, q, r\right)\right) \mathrm{d} F_{-1}}{\int_{v_{-1}} f_{1}\left(\widetilde{v}_{1}\left(v_{-1} ; p^{*}, q, r\right)\right) \mathrm{d} F_{-1}}\left(r_{1}-q_{1}\right) \\
p_{B}^{*}-C(r)= & \frac{\int_{v_{-1}}\left[1-F_{1}\left(\widetilde{v}_{1}\left(v_{-1} ; p^{*}, q, r\right)\right)\right] \mathrm{d} F_{-1}}{\int_{v_{-1}} f_{1}\left(\widetilde{v}_{1}\left(v_{-1} ; p^{*}, q, r\right)\right) \mathrm{d} F_{-1}}\left(r_{1}-q_{1}\right)
\end{aligned}
$$

where

$$
\widetilde{v}_{1}\left(v_{-1} ; p^{*}, q, r\right)=\frac{p_{B}^{*}-p_{A}^{*}}{r_{1}-q_{1}}-\sum_{k=2}^{N} v_{k} \frac{r_{k}-q_{k}}{r_{1}-q_{1}}, \quad v_{k} \in\left[\underline{v}_{k}, \bar{v}_{k}\right], \quad k=2, \ldots, N
$$

Equations (6) and (7) say that the price-cost margins follow the usual inverse elasticity rule, a standard result. ${ }^{5}$ The complication is that (8) sets up a function $\widetilde{v}_{1}$ that depends on $N-1$ continuous variables $v_{k}$, $k=2, . ., N$, and the quality vector $(q, r)$, and this is to be determined simultaneously with the prices in $(6)$ and (7).

Let $p^{*}=\left(p_{A}^{*}, p_{B}^{*}\right)$ be the subgame-perfect equilibrium prices in Stage 2 in subgame $(q, r)$. The equilibrium prices are functions of the quality vector, so we write them as $p^{*}(q, r)$. Let $\widetilde{v}_{1}\left(v_{-1} ; p^{*}(q, r), q, r\right)$ be the solution of (8) at the subgame-perfect equilibrium. Now we define $\widetilde{v}_{1}^{*}\left(v_{-1} ; q, r\right) \equiv \widetilde{v}_{1}\left(v_{-1} ; p^{*}(q, r), q, r\right)$, which describes the set of consumers who are indifferent between buying from Firm $A$ and Firm $B$ in an equilibrium in subgame $(q, r)$. By substituting the equilibrium prices $(6)$ and $(7)$ into $\widetilde{v}_{1}\left(v_{-1} ; p^{*}(q, r), q, r\right)$ in (8) above, we have:

$$
\widetilde{v}_{1}^{*}\left(v_{-1} ; q, r\right)=\frac{\int_{v_{-1}}\left[1-2 F_{1}\left(\widetilde{v}_{1}^{*}\left(v_{-1} ; q, r\right)\right)\right] \mathrm{d} F_{-1}}{\int_{v_{-1}} f_{1}\left(\widetilde{v}_{1}^{*}\left(v_{-1} ; q, r\right)\right) \mathrm{d} F_{-1}}+\frac{C(r)-C(q)}{r_{1}-q_{1}}-\sum_{k=2}^{N} v_{k} \frac{r_{k}-q_{k}}{r_{1}-q_{1}} .
$$

This is an integral equation in $\widetilde{v}_{1}^{*}$, and the solution holds the key to the characterization of the price equilibrium. Indeed, we have decomposed the system in (6), (7) and (8) into two systems: a single integral equation (9), and those two equations (6) and (7). The integral equation (9) is independent of prices. Using the solution to (9), we can then proceed to solve for the equilibrium prices in (6) and (7). We are unaware

\footnotetext{
${ }^{5}$ If we divide (6) by $p_{A}^{*}$, it can easily be seen that the right-hand side is the inverse elasticity of demand, which is obtained from the demand $\int_{v_{-1}} F_{1}\left(\widetilde{v}_{1}\left(v_{-1} ; p, q, r\right)\right) \mathrm{d} F_{-1}$.
} 
that any paper in the extant literature of multiple qualities has decomposed the equilibrium prices and demand characterization in this fashion. Yet, using this decomposition, we can characterize the functional relationship between quality and equilibrium prices.

Lemma 1 The solution of the integral equation (9) takes the form $\widetilde{v}_{1}^{*}\left(v_{-1} ; q, r\right)=\alpha(q, r)-\sum_{k=2}^{N} v_{k} \beta_{k}(q, r)$, for $v_{k} \in\left[\underline{v}_{k}, \bar{v}_{k}\right], k=2, \ldots, N$, where the functions $\alpha$ and $\beta_{k}$ are defined by

$$
\begin{aligned}
\alpha(q, r) & =\frac{\int_{v_{-1}}\left[1-2 F_{1}\left(\alpha(q, r)-\sum_{k=2}^{N} v_{k} \beta_{k}(q, r)\right)\right] d F_{-1}}{\int_{v_{-1}} f_{1}\left(\alpha(q, r)-\sum_{k=2}^{N} v_{k} \beta_{k}(q, r)\right) d F_{-1}}+\frac{C(r)-C(q)}{r_{1}-q_{1}} \\
\beta_{k}(q, r) & =\frac{r_{k}-q_{k}}{r_{1}-q_{1}}, \quad k=2, \ldots, N .
\end{aligned}
$$

The solution in Lemma $1, \widetilde{v}_{1}^{*}\left(v_{-1} ; q, r\right)=\alpha(q, r)-v_{-1} \cdot \beta_{-1}(q, r)$, describes the equilibrium set of indifferent consumers in subgame $(q, r)$ where we have used the common abbreviation $\beta_{-1}(q, r) \equiv\left(\beta_{2}(q, r), \beta_{3}(q, r)\right.$, $\left.\ldots, \beta_{N}(q, r)\right)$. From (6) and (7), a firm's qualities affect equilibrium prices of both firms. In turn, when equilibrium prices change, the set of indifferent consumers change accordingly. The composition of the quality effect on equilibrium prices, and then the effect of equilibrium prices on the equilibrium set of indifferent consumers is the solution in Lemma 1. The equilibrium set of indifferent consumers takes the linear form, so the intercept $\alpha$ and all the slopes $\beta_{k}, k=2, \ldots, N$ are functions of the qualities.

Lemma 1 is a remarkable result. First, the solution of the integral equation (9) takes a manageable form: it consists of one implicit function $\alpha(q, r)$ in (10) and $N-1$ explicit (and simple) functions $\beta_{k}(q, r)$, $k=2, \ldots, N$, in (11). In other words, for any fixed number of quality attributes, there is only one implicit function like the one in (10) to handle. Also, the equilibrium prices boil down to solving for the solutions of just three equations. From the discussion above and by substituting the expressions for (10) and (11) to the right-hand side of (6) and (7), we can state the following proposition (proof omitted): 
Proposition 1 In subgame $(q, r)$, equilibrium prices are the solution of $p_{A}^{*}$ in (12) and $p_{B}^{*}$ in (13):

$$
\begin{aligned}
p_{A}^{*}-C(q)= & \frac{\int_{v_{-1}} F_{1}\left(\alpha(q, r)-v_{-1} \cdot \beta_{-1}(q, r)\right) d F_{-1}}{\int_{v_{-1}} f_{1}\left(\alpha(q, r)-v_{-1} \cdot \beta_{-1}(q, r)\right) d F_{-1}}\left(r_{1}-q_{1}\right) \\
p_{B}^{*}-C(r)= & \frac{\int_{v_{-1}}\left[1-F_{1}\left(\alpha(q, r)-v_{-1} \cdot \beta_{-1}(q, r)\right)\right] d F_{-1}}{\int_{v_{-1}} f_{1}\left(\alpha(q, r)-v_{-1} \cdot \beta_{-1}(q, r)\right) d F_{-1}}\left(r_{1}-q_{1}\right),
\end{aligned}
$$

with $\alpha(q, r)$ implicitly defined by (10), and $\beta_{k}(q, r)=\frac{r_{k}-q_{k}}{r_{1}-q_{1}}, k=2, \ldots, N$.

The importance of Proposition 1 is this. The equilibrium price $p_{A}^{*}$ is given by (12), an explicit function of qualities. Thus, a direct differentiation of $p_{A}^{*}$ with respect to qualities yields all the relevant information of how any of Firm $B$ 's quality choice changes Firm $A$ 's equilibrium price. The same applies to $p_{B}^{*}$ and (13). The common link between $p_{A}^{*}$ in (12) and $p_{B}^{*}$ in (13) is the implicit function (10), the explicit functions (11), and the distributions of quality valuations. How do qualities change prices?

\subsection{Qualities and equilibrium prices}

We begin with writing equilibrium prices $p_{A}^{*}$ in (12) and $p_{B}^{*}$ in (13) as

$$
\frac{p_{A}^{*}-C(q)}{r_{1}-q_{1}}=G\left(\alpha, \beta_{-1}\right) \quad \text { and } \quad \frac{p_{B}^{*}-C(r)}{r_{1}-q_{1}}=H\left(\alpha, \beta_{-1}\right)
$$

where the functions: $G\left(\alpha, \beta_{-1}\right): \Re^{N} \rightarrow \Re$, and $H\left(\alpha, \beta_{-1}\right): \Re^{N} \rightarrow \Re$ are defined by

$$
\begin{aligned}
G\left(\alpha, \beta_{-1}\right) & \equiv \frac{\int_{v_{-1}} F_{1}\left(\alpha-v_{-1} \cdot \beta_{-1}\right) \mathrm{d} F_{-1}}{\int_{v_{-1}} f_{1}\left(\alpha-v_{-1} \cdot \beta_{-1}\right) \mathrm{d} F_{-1}} \\
H\left(\alpha, \beta_{-1}\right) & \equiv \frac{\int_{v_{-1}}\left[1-F_{1}\left(\alpha-v_{-1} \cdot \beta_{-1}\right)\right] \mathrm{d} F_{-1}}{\int_{v_{-1}} f_{1}\left(\alpha-v_{-1} \cdot \beta_{-1}\right) \mathrm{d} F_{-1}} .
\end{aligned}
$$

The functions $G$ and $H$ are the firms' price-cost markups per unit of quality difference. Working directly with these price-cost markups has afforded us tractability. ${ }^{6}$ However, our final results will be expressed in

\footnotetext{
${ }^{6}$ For a model with only one quality attribute, the markups are the reverse hazard rate, $F / f$, and the hazard rate $(1-F) / f$, where $F$ and $f$ are the distribution and density functions of the quality valuation.
} 
terms of the primitives of the model: the density and distribution functions, $f_{i}$ and $F_{i}, i=1,2 \ldots, N$ and the cost function $C$. We have omitted the quality arguments in $\alpha$ and $\beta_{-1} \equiv\left(\beta_{2}, \ldots, \beta_{N}\right)$ for a shorter set of notation here. The numerators of $G\left(\alpha, \beta_{-1}\right)$ and $H\left(\alpha, \beta_{-1}\right)$ are, respectively, Firm $A$ 's and Firm $B$ 's demands. The common denominator is the total density of the set of indifferent consumers. Indifferent consumers are those with valuations satisfying $v \cdot q-p_{A}=v \cdot r-p_{B}$. Hence, the total density of this set is $\int_{v \cdot q-p_{A}=v \cdot r-p_{B}} \prod_{i=1}^{N} \mathrm{~d} F_{i}\left(v_{i}\right)=\int_{v_{-1}} f_{1}\left(\widetilde{v}_{1}^{*}\right) \mathrm{d} F_{-1}$.

Recall that $\alpha(q, r)$ and $\beta_{k}(q, r)$ are given by Lemma 1 . Hence, we can directly differentiate $p_{A}^{*}$ with respect to Firm $B$ 's qualities, and differentiate $p_{B}^{*}$ with respect Firm $A$ 's qualities, and we call these the price-reaction effects:

$$
\begin{aligned}
& \frac{\partial p_{A}^{*}}{\partial r_{1}}=\left(r_{1}-q_{1}\right) \frac{\partial G\left(\alpha, \beta_{-1}\right)}{\partial r_{1}}=G\left(\alpha, \beta_{-1}\right)+\left(r_{1}-q_{1}\right)\left[\frac{\partial G}{\partial \alpha} \frac{\partial \alpha}{\partial r_{1}}+\sum_{k=2}^{N} \frac{\partial G}{\partial \beta_{k}} \frac{\partial \beta_{k}}{\partial r_{1}}\right] \\
& \frac{\partial p_{B}^{*}}{\partial q_{1}}=\left(r_{1}-q_{1}\right) \frac{\partial H\left(\alpha, \beta_{-1}\right)}{\partial q_{1}}=-H\left(\alpha, \beta_{-1}\right)+\left(r_{1}-q_{1}\right)\left[\frac{\partial H}{\partial \alpha} \frac{\partial \alpha}{\partial q_{1}}+\sum_{k=2}^{N} \frac{\partial H}{\partial \beta_{k}} \frac{\partial \beta_{k}}{\partial q_{1}}\right],
\end{aligned}
$$

and

$$
\begin{array}{ll}
\frac{\partial p_{A}^{*}}{\partial r_{j}}=\left(r_{1}-q_{1}\right) \frac{\partial G\left(\alpha, \beta_{-1}\right)}{\partial r_{j}}=\left(r_{1}-q_{1}\right)\left[\frac{\partial G}{\partial \alpha} \frac{\partial \alpha}{\partial r_{j}}+\frac{\partial G}{\partial \beta_{j}} \frac{\partial \beta_{j}}{\partial r_{j}}\right], \quad j=2, \ldots, N \\
\frac{\partial p_{B}^{*}}{\partial q_{j}}=\left(r_{1}-q_{1}\right) \frac{\partial H\left(\alpha, \beta_{-1}\right)}{\partial q_{j}}=\left(r_{1}-q_{1}\right)\left[\frac{\partial H}{\partial \alpha} \frac{\partial \alpha}{\partial q_{j}}+\frac{\partial H}{\partial \beta_{j}} \frac{\partial \beta_{j}}{\partial q_{j}}\right], \quad j=2, \ldots, N .
\end{array}
$$

Because we label a differentiated quality attribute as the first attribute $\left(q_{1}<r_{1}\right)$, there is a slight difference between the form of price-reaction effects of the first quality and the other qualities. (With all the arguments displayed, the function $G$ is $G\left(\alpha, \beta_{2}, \ldots, \beta_{N}\right)=G\left(\alpha, \frac{r_{2}-q_{2}}{r_{1}-q_{1}}, \ldots, \frac{r_{N}-q_{N}}{r_{1}-q_{1}}\right)$. Similarly, for the function $H$. The arguments $\beta_{2}, \ldots, \beta_{N}$ in $G$ and $H$ include $r_{1}$ and $q_{1}$, so if we partially differentiate $G$ or $H$ with respect to $r_{1}$ and $q_{1}$, we get the summation terms in (16) and (17). However, for $j=2, \ldots, N, r_{j}$ and $q_{j}$ are only in the $\operatorname{argument} \beta_{j}$ in $G$ and $H$. When we partially differentiate $G$ or $H$ with respect to $r_{j}$ or $q_{j}$, we only get the simpler expressions in (18) and (19).)

The expression in (18) displays a composition of two effects. The first is how qualities change the intercept $\alpha$ and slopes $\beta_{-1}$ of the equation that determines the equilibrium set of indifferent consumers 
$\widetilde{v}_{1}^{*}\left(v_{-1} ; q, r\right)=\alpha(q, r)-v_{-1} \cdot \beta_{-1}(q, r)$; these are the terms $\frac{\partial \alpha}{\partial r_{j}}, \frac{\partial \beta_{j}}{\partial r_{j}}, \frac{\partial \alpha}{\partial q_{j}}$, and $\frac{\partial \beta_{j}}{\partial q_{j}}$. The second is how the equilibrium set of consumers change the price-cost markups $G\left(\alpha, \beta_{-1}\right)$ and $H\left(\alpha, \beta_{-1}\right)$; these are the terms $\frac{\partial G}{\partial \alpha}, \frac{\partial G}{\partial \beta_{j}}, \frac{\partial H}{\partial \alpha}$ and $\frac{\partial H}{\partial \beta_{j}}, j=2, \ldots, N$

We first present how the sum in markups varies with the equilibrium set of indifferent consumers. Then we can obtain how the sum varies with the intercept and slope of the equations for the set of indifferent consumers.

Lemma 2 In any subgame $(q, r)$, the sum of the proportional changes in the firms' equilibrium price-cost markups and the proportional change in the total density of the equilibrium set of indifferent consumers must vanish:

$$
d \ln \left[G\left(\alpha, \beta_{-1}\right)+H\left(\alpha, \beta_{-1}\right)\right]+d \ln \int_{v_{-1}} f_{1}\left(\alpha-v_{-1} \cdot \beta_{-1}\right) d F_{-1}=0 .
$$

It follows that the sum of the partial derivatives of $G\left(\alpha, \beta_{-1}\right)$ and $H\left(\alpha, \beta_{-1}\right)$ with respect to $\alpha$ and $\beta_{j}$, $j=2, \ldots, N$ are

$$
\begin{gathered}
\frac{\partial G}{\partial \alpha}+\frac{\partial H}{\partial \alpha}=-\frac{\int_{v_{-1}} f_{1}^{\prime}\left(\alpha-v_{-1} \cdot \beta_{-1}\right) d F_{-1}}{\left(\int_{v_{-1}} f_{1}\left(\alpha-v_{-1} \cdot \beta_{-1}\right) d F_{-1}\right)^{2}} \\
\frac{\partial G}{\partial \beta_{j}}+\frac{\partial H}{\partial \beta_{j}}=\frac{\int_{v_{-1}} f_{1}^{\prime}\left(\alpha-v_{-1} \cdot \beta_{-1}\right) v_{j} d F_{-1}}{\left(\int_{v_{-1}} f_{1}\left(\alpha-v_{-1} \cdot \beta_{-1}\right) d F_{-1}\right)^{2}} .
\end{gathered}
$$

Lemma 2 follows from the definitions of $G$ and $H$ and from straightforward differentiation. From the definitions (14) and (15), we have:

$$
G+H=\frac{1}{\int_{v_{-1}} f_{1}\left(\alpha-v_{-1} \cdot \beta_{-1}\right) \mathrm{d} F_{-1}} .
$$

At each subgame $(q, r)$ the market is always covered, the total price-cost markup is always equal to the reciprocal of the total density of equilibrium set of indifferent consumes. Hence, equation (20) follows. If somehow the intercept of the equilibrium set of consumers $(\alpha)$ increases, the sum of firms' price-cost markups 
will be in (21). Similarly, if the $j^{\text {th }}$ slope of the equilibrium set of indifferent consumers $\left(\beta_{j}\right)$ increases, the sum of their markups will be in (22). Next, we present how qualities change the intercept and slopes of the equation for the equilibrium set of indifferent consumers.

Lemma 3 In any subgame $(q, r)$, in equilibrium, for $i=1, \ldots, N$ and $j=2, \ldots, N$,

$$
\frac{\partial \alpha(q, r)}{\partial q_{i}}+\frac{\partial \alpha(q, r)}{\partial r_{i}}=\frac{C_{j}(r)-C_{j}(q)}{\left(r_{1}-q_{1}\right)\left[1+\frac{\partial G}{\partial \alpha}-\frac{\partial H}{\partial \alpha}\right]} \quad \text { and } \quad \frac{\partial \beta_{j}(q, r)}{\partial q_{i}}+\frac{\partial \beta_{j}(q, r)}{\partial r_{i}}=0
$$

The continuation price equilibrium of subgame $(q, r)$ generates the equilibrium set of indifference consumers, $\widetilde{v}_{1}^{*}\left(v_{-1} ; q, r\right)=\alpha(q, r)-v_{-1} \cdot \beta_{-1}(q, r)$. Firms changing their qualities will impact the equilibrium prices of both firms, and the equilibrium set of indifferent consumers. Intercept $\alpha(q, r)$ and slopes $\beta_{k}(q, r)$ will be changed by the change in $q_{i}$. Lemma 3 describes some relations of how qualities change the intercept and slopes. The sum of the effects of firms' quality changes on the intercept is proportional to the difference in marginal cost. The sum of the effects of firms' quality changes on the slope is zero.

Lemmas 3 can be used to characterize firms' relative strength of strategic price effects.

Proposition 2 In subgame $(q, r)$, for quality $j, j=2, \ldots, N$, the difference in the strategic price effects, 


$$
\begin{aligned}
& \frac{\partial p_{B}^{*}(q, r)}{\partial q_{j}}-\frac{\partial p_{A}^{*}(q, r)}{\partial r_{j}} \text {, can be written in three equivalent forms: } \\
& \frac{\left(r_{1}-q_{1}\right)\left[\begin{array}{c}
\int_{v_{-1}} f_{1}^{\prime}\left(\alpha-v_{-1} \cdot \beta_{-1}\right) d F_{-1} \frac{\partial \alpha}{\partial r_{j}} \\
-\int_{v_{-1}} f_{1}^{\prime}\left(\alpha-v_{-1} \cdot \beta_{-1}\right) v_{k} d F_{-1} \frac{\partial \beta_{j}}{\partial r_{j}}
\end{array}\right]}{\left(\int_{v_{-1}} f_{1}\left(\alpha-v_{-1} \cdot \beta_{-1}\right) d F_{-1}\right)^{2}}+Z\left[C_{j}(q)-C_{j}(r)\right] \\
& =\frac{\left(r_{1}-q_{1}\right) \frac{\partial}{\partial r_{j}}\left[\int_{v_{-1}} f_{1}\left(\alpha-v_{-1} \cdot \beta_{-1}\right) d F_{-1}\right]}{\left(\int_{v_{-1}} f_{1}\left(\alpha-v_{-1} \cdot \beta_{-1}\right) d F_{-1}\right)^{2}}+Z\left[C_{j}(q)-C_{j}(r)\right] \\
& =\frac{-\left(r_{1}-q_{1}\right) \frac{\partial}{\partial q_{j}}\left[\int_{v_{-1}} f_{1}\left(\alpha-v_{-1} \cdot \beta_{-1}\right) d F_{-1}\right]}{\left(\int_{v_{-1}} f_{1}\left(\alpha-v_{-1} \cdot \beta_{-1}\right) d F_{-1}\right)^{2}}+Z\left[C_{j}(q)-C_{j}(r)\right], \\
& \text { where } Z=\left\{\frac{1+H\left(\alpha, \beta_{-1}\right) \frac{\int_{v_{-1}} f_{1}^{\prime}\left(\alpha-v_{-1} \cdot \beta_{-1}\right) d F_{-1}}{\int_{v_{-1}} f_{1}\left(\alpha-v_{-1} \cdot \beta_{-1}\right) d F_{-1}}}{3-G\left(\alpha, \beta_{-1}\right) \frac{\int_{v_{-1}} f_{1}^{\prime}\left(\alpha-v_{-1} \cdot \beta_{-1}\right) d F_{-1}}{\int_{v_{-1}} f_{1}\left(\alpha-v_{-1} \cdot \beta_{-1}\right) d F_{-1}}}\right\} \text {. }
\end{aligned}
$$

The proposition says that the difference in firms' strategic price effects of a quality is determined by how the quality changes the total density of the equilibrium set of indifferent consumers, $\int_{v_{-1}} f_{1}\left(\alpha-v_{-1} \cdot \beta_{-1}\right) \mathrm{d} F_{-1}$, and by the difference in the marginal cost of quality $\left[C_{j}(q)-C_{j}(r)\right]$. The sum of the functions $G$ and $H$ was written in (23). The first term in each of the equivalent expressions in the Proposition is the derivative of the sum of $G$ and $H$ with respect to a quality $j, j=2, \ldots, N$, multiplied by $\left(r_{1}-q_{1}\right)$. In fact, the effect of Firm A's quality on the total density of the set of indifferent consumers is equal and opposite of that of Firm B's. The second term is the difference in firms' marginal costs of a quality adjusted by $Z$.

Finally, we note that when $f_{1}$ is a step function, $f_{1}^{\prime}\left(\alpha-v_{-1} \cdot \beta_{-1}\right)=0$, expressions from (25) to (27) 
simplify to only the term related to the difference in marginal costs:

$$
\frac{\partial p_{B}^{*}(q, r)}{\partial q_{j}}-\frac{\partial p_{A}^{*}(q, r)}{\partial r_{j}}=\frac{1}{3}\left[C_{j}(q)-C_{j}(r)\right]
$$

In other words, when the differentiated dimension has a uniform-distribution valuation, each firm's quality raises the markup by the same amount, so the strategic price reaction effects are all in the marginal-cost difference.

\subsection{Equilibrium qualities}

Given the equilibrium prices $p_{A}^{*}(q, r)$ and $p_{B}^{*}(q, r)$ in Stage 2, we write the profit functions in Stage 1 in terms of qualities:

$$
\begin{aligned}
& \pi_{A}\left(p_{A}^{*}(q, r), p_{B}^{*}(q, r) ; q, r\right)=\int_{v_{-1}} F_{1}\left(\widetilde{v}_{1}\left(v_{-1} ; p^{*}, q, r\right)\right) \mathrm{d} F_{-1}\left[p_{A}^{*}(q, r)-C(q)\right] \\
& \pi_{B}\left(p_{A}^{*}(q, r), p_{B}^{*}(q, r) ; q, r\right)=\int_{v_{-1}}\left[1-F_{1}\left(\widetilde{v}_{1}\left(v_{-1} ; p^{*}, q, r\right)\right)\right] \mathrm{d} F_{-1}\left[p_{B}^{*}(q, r)-C(r)\right],
\end{aligned}
$$

where $\widetilde{v}_{1}\left(v_{-1} ; p^{*}, q, r\right) \equiv \frac{p_{B}^{*}(q, r)-p_{A}^{*}(q, r)}{r_{1}-q_{1}}-\sum_{k=2}^{N} v_{k} \frac{r_{k}-q_{k}}{r_{1}-q_{1}}$. For subgame-perfect equilibrium prices, $p^{*}$, equilibrium qualities are $q^{*}$ and $r^{*}$ that are mutual best responses:

$$
\begin{aligned}
q^{*} & \equiv\left(q_{1}^{*}, \ldots, q_{N}^{*}\right)=\underset{q}{\operatorname{argmax}} \int_{v_{-1}} F_{1}\left(\widetilde{v}_{1}\left(v_{-1} ; p^{*}\left(q, r^{*}\right), q, r^{*}\right)\right) \mathrm{d} F_{-1}\left[p_{A}^{*}\left(q, r^{*}\right)-C(q)\right] \\
r^{*} & \equiv\left(r_{1}^{*}, \ldots, r_{N}^{*}\right)=\underset{r}{\operatorname{argmax}} \int_{v_{-1}}\left[1-F_{1}\left(\widetilde{v}_{1}\left(v_{-1} ; p^{*}\left(q^{*}, r\right), q^{*}, r\right)\right)\right] \mathrm{d} F_{-1}\left[p_{B}^{*}\left(q^{*}, r\right)-C(r)\right],
\end{aligned}
$$

where $p^{*}(q, r) \equiv\left(p_{A}^{*}(q, r), p_{B}^{*}(q, r)\right)$.

Qualities $q_{i}, i=1, \ldots, N$ affect Firm $A$ 's profit (28) in three ways. First, they have a direct effect through the costs, $C(q)$, as well as the demand. Second, they affect the profit through Firm A's own equilibrium price $p_{A}^{*}(q, r)$. Third, they affect the profit through Firm $B$ 's equilibrium price $p_{B}^{*}(q, r)$, captured by $\partial p_{B}^{*} / \partial q_{i}$. Because the equilibrium prices $p_{A}^{*}(q, r)$ and $p_{B}^{*}(q, r)$ are mutual best responses in the price subgame in Stage 2 , the envelope theorem applies. That is, Firm $A$ 's qualities $q_{i}, i=1, \ldots, N$ have second-order effects on its own profit (28) through its equilibrium price; the second effect can be ignored. 
The first-order derivative of (28) with respect to $q_{i}$ is

$$
\begin{gathered}
-\left[\int_{v_{-1}} F_{1}\left(\widetilde{v}_{1}\left(v_{-1} ; p^{*}\left(q, r^{*}\right), q, r^{*}\right)\right) \mathrm{d} F_{-1}\right] C_{i}(q) \\
+\underbrace{\frac{\partial}{\partial q_{i}} \int_{v_{-1}} F_{1}\left(\widetilde{v}_{1}\left(v_{-1} ; p^{*}\left(q, r^{*}\right), q, r^{*}\right)\right) \mathrm{d} F_{-1}\left[p_{A}^{*}\left(q, r^{*}\right)-C(q)\right]}_{\text {effects of quality } q_{i} \text { on cost and demand }} \\
+\underbrace{\frac{\partial}{\partial p_{B}^{*}}\left\{\int_{v_{-1}} F_{1}\left(\widetilde{v}_{1}\left(v_{-1} ; p^{*}\left(q, r^{*}\right), q, r^{*}\right)\right) \mathrm{d} F_{-1}\right\} \frac{\partial p_{B}^{*}}{\partial q_{i}}\left[p_{A}^{*}\left(q, r^{*}\right)-C(q)\right]}_{\text {effect of quality on Firm } B^{\prime} \text { s price }}, \quad i=1, \ldots, N,
\end{gathered}
$$

where the (partial) derivative of profit with respect to $p_{A}$ has been ignored. The terms in (30) describe how a quality affects cost and demand, whereas the term in (31) describes the strategic effect of a quality on the rival's price.

Next, we use the same steps to obtain the first-order derivatives of Firm B's profit (29) with respect to $r_{i}$, and these are

$$
\begin{gathered}
-\left[\int_{v_{-1}}\left[1-F_{1}\left(\widetilde{v}_{1}\left(v_{-1} ; p^{*}\left(q^{*}, r\right), q^{*}, r\right)\right)\right] \mathrm{d} F_{-1}\right] C_{i}(r) \\
+\underbrace{\frac{\partial}{\partial r_{i}}\left\{\int_{v_{-1}}\left[1-F_{1}\left(\widetilde{v}_{1}\left(v_{-1} ; p^{*}\left(q^{*}, r\right), q^{*}, r\right)\right)\right] \mathrm{d} F_{-1}\right\}\left[p_{B}^{*}\left(q^{*}, r\right)-C(r)\right]}_{\text {effects of quality on cost and demand }} \\
+\underbrace{\frac{\partial}{\partial p_{A}^{*}}\left\{\int_{v_{-1}}\left[1-F_{1}\left(\widetilde{v}_{1}\left(v_{-1} ; p^{*}\left(q^{*}, r\right), q^{*}, r\right)\right)\right] \mathrm{d} F_{-1}\right\} \frac{\partial p_{A}^{*}}{\partial r_{i}}\left[p_{A}^{*}\left(q, r^{*}\right)-C(r)\right]}_{\text {effect of quality } r_{i} \text { on Firm } A^{\prime} \text { s price }}, \quad i=1, \ldots, N .
\end{gathered}
$$

These expressions carry the same interpretations as those in the first-order derivatives for Firm $A$.

We now state the main result on equilibrium qualities. We obtain the set of equations in the next proposition by first simplifying the first-order derivatives and then setting them to zero. For simplification, we use the basic demand function (3) and equilibrium prices (12) and (13) in Proposition 1, and finally drop common factors in the first-order derivatives. (Details are in the proof.)

Proposition 3 For the quality-price, multistage game in Subsection 2.2, equilibrium qualities $\left(q^{*}, r^{*}\right)$ (under 
the convention that $\left.q_{1}^{*}<r_{1}^{*}\right)$ must satisfy the following $2 N$ equations:

$$
\begin{aligned}
& \frac{\partial p_{B}^{*}}{\partial q_{1}}+\frac{\int_{v_{-1}} f_{1}\left(\alpha-v_{-1} \cdot \beta_{-1}\right) \widetilde{v}_{1}^{*} d F_{-1}}{\left.\int_{v_{-1}} f_{1}\left(\alpha-v_{-1} \cdot \beta_{-1}\right)\right) d F_{-1}}-C_{1}\left(q^{*}\right)=0 \\
& \frac{\partial p_{A}^{*}}{\partial r_{1}}+\frac{\int_{v_{-1}} f_{1}\left(\alpha-v_{-1} \cdot \beta_{-1}\right) \widetilde{v}_{1}^{*} d F_{-1}}{\left.\int_{v_{-1}} f_{1}\left(\alpha-v_{-1} \cdot \beta_{-1}\right)\right) d F_{-1}}-C_{1}\left(r^{*}\right)=0
\end{aligned}
$$

and for $j=2, \ldots, N$,

$$
\begin{aligned}
& \frac{\partial p_{B}^{*}}{\partial q_{j}}+\frac{\int_{v_{-1}} f_{1}\left(\alpha-v_{-1} \cdot \beta_{-1}\right) v_{j} d F_{-1}}{\left.\int_{v_{-1}} f_{1}\left(\alpha-v_{-1} \cdot \beta_{-1}\right)\right) d F_{-1}}-C_{j}\left(q^{*}\right)=0 \\
& \frac{\partial p_{A}^{*}}{\partial r_{j}}+\frac{\int_{v_{-1}} f_{1}\left(\alpha-v_{-1} \cdot \beta_{-1}\right) v_{j} d F_{-1}}{\left.\int_{v_{-1}} f_{1}\left(\alpha-v_{-1} \cdot \beta_{-1}\right)\right) d F_{-1}}-C_{j}\left(r^{*}\right)=0 .
\end{aligned}
$$

where $\alpha$ and $\beta_{j}$ are the functions in (10) and (11), respectively, and $\widetilde{v}_{1}^{*}$ is $\widetilde{v}_{1}^{*}\left(v_{-1} ; q^{*}, r^{*}\right)$, the solution of the integral equation in Lemma 1.

The properties of equilibrium qualities in (34) and (36) can be explained as follows. There are two effects. The first term in each expression is the price-reaction effect: it describes how Firm A's qualities $q_{1}$ and $q_{j}$, $j=2, \ldots, N$ affect the rival's price in the continuation subgame. In fact, all the price-reaction effects are written out in equations (16) to (19) above.

The second effect concerns the average valuation of the $j^{\text {th }}$ quality among the equilibrium set of indifferent consumers - the integrals in (34) and (36) - and the $j^{\text {th }}$ quality's marginal contribution to the per-unit cost $-C_{j} \equiv \frac{\partial C(q)}{\partial q_{j}}$. These two terms together form the Spence effect. Indeed, Spence (1975) shows that a profit-maximizing firm chooses the efficient quality for the marginal consumer (and then raises the price to extract the marginal consumer's surplus). ${ }^{7}$ The same price-reaction and Spence effects apply to Firm $B$ 's equilibrium quality choices described by (35) and (37).

${ }^{7}$ Let $P(D, q)$ be the price a firm can charge when it sells $D$ units of its good at quality $q=\left(q_{1}, \ldots, q_{N}\right)$. Let $C(D, q)$ be the cost when the firm produces $D$ units at quality $q$. Profit is $D P(D, q)-C(D, q)$. The profit-maximizing quality $q_{i}$ is given by $D \frac{\partial P}{\partial q_{i}}=\frac{\partial C}{\partial q_{i}}$. Hence the quality valuation of the marginal consumer $\frac{\partial P}{\partial q_{i}}$ is equal to the marginal contribution of quality $i$ to per-unit cost $\frac{\partial C / \partial q_{i}}{D}$. See Spence $(1975$, p419; equation (8)). 
Because the two firms face the same equilibrium set of indifferent consumers, the Spence effect pushes them to choose the same qualities. The price-reaction effects generally put the firms in a race situation. Prices are strategic complements, so each firm wants to use its qualities to raise the rival's price. The price-reaction effect dictates how much a firm's equilibrium quality deviates from the efficient quality for the equilibrium set of indifferent consumers. The firm that has a stronger strategic price-reaction effect deviates more.

If the two firms were playing another game in which prices and qualities were chosen concurrently (one with merged Stages 1 and 2 in the extensive form in Subsection 2.2), the price-reaction effect would vanish. Then the Spence effect would dictate equilibrium strategies. Each firm would choose the qualities optimal for the average valuations of the common set of marginal consumers, so firms choose the same level for each quality attribute. Firms must then set their prices at marginal cost. (For an illustration of a game with firms choosing prices and qualities concurrently, see Ma and Burgess (1993)).

Proposition 3 holds the key to the understanding of equilibrium product differentiation with multiple qualities, to which we now turn.

\subsection{Quality differentiation}

Proposition 3 draws a connection between the price-reaction effects and qualities' marginal contributions to unit production cost. We state this formally:

Corollary 1 At the equilibrium $\left(q^{*}, r^{*}\right)$, a firm's $j^{\text {th }}$ quality contributes more to its own unit production cost than a rival's $j^{\text {th }}$ quality contributes to the rival's unit production cost if and only if the firm's price-reaction effect of that quality is stronger than the rival's. That is, for each $j=2, \ldots, N$, the following are equivalent:

i) $C_{j}\left(q^{*}\right)<C_{j}\left(r^{*}\right)$,

ii) $\frac{\partial p_{B}^{*}\left(q^{*}, r^{*}\right)}{\partial q_{j}}<\frac{\partial p_{A}^{*}\left(q^{*}, r^{*}\right)}{\partial r_{j}}$,

iii) the three equivalent expressions (25), (26), and (27) in Proposition 2 are negative at equilibrium $\left(q^{*}, r^{*}\right)$.

Corollary 1 follows from Proposition 3 straightfowardly; we simply take the difference between first-order conditions. On the cost side, the fundamental issue is how much a quality contributes to the unit production 
cost. On the strategic side, the fundamental issue is how much a quality affects the rival's price. The quality race concerns only these two issues. However, the corollary does not directly address the equilibrium quality levels. We have used a general cost function, so it is quite possible that $C_{j}\left(q^{*}\right)<C_{j}\left(r^{*}\right)$ but $q_{j}^{*}>r_{j}^{*}$. (For an illustration, see the example after Corollary 3.) Sharper results can be obtained from the following (with proof omitted):

Corollary 2 Suppose that the cost function $C$ is separable:

$$
C(q)=C\left(q_{1}, q_{2}, \ldots, q_{N}\right)=\gamma_{1}\left(q_{1}\right)+\gamma_{2}\left(q_{2}\right), \ldots \gamma_{N}\left(q_{N}\right)
$$

where $\gamma_{i}$ is an increasing and convex function, so $C_{i}(q)=\gamma_{i}^{\prime}\left(q_{i}\right), i=1,2, \ldots, N$. In an equilibrium $\left(q^{*}, r^{*}\right)$, for $j=2, \ldots, N$,

$$
q_{j}^{*}<r_{j}^{*} \Longleftrightarrow \frac{\partial p_{B}^{*}\left(q^{*}, r^{*}\right)}{\partial q_{j}}<\frac{\partial p_{A}^{*}\left(q^{*}, r^{*}\right)}{\partial r_{j}}
$$

With separable cost, a quality's contribution to the unit production cost is independent of other qualities. A firm having a stronger price-reaction effect at a quality than its rival's must choose a higher quality than its rival's quality. In the literature, the separable cost function has been adopted. Corollaries 1 and 2 can be read as a caution. The fundamental issue is how a quality contributes to the production cost. For a model with many qualities, a quality's contribution to production cost depends on the entire vector of qualities. Corollaries 1 and 2 together say that such interdependence is important; see also the example following the next result.

Next, we consider specific quality valuation density functions commonly used in the literature. Recall that, with a step function the difference of the price effects reduces to $\frac{1}{3}\left[C_{j}(q)-C_{j}(r)\right]$. Hence:

Corollary 3 Suppose that $f_{1}$ is a step function, so $f_{1}^{\prime}=0$ almost everywhere. In an equilibrium $\left(q^{*}, r^{*}\right)$, $C_{j}\left(q^{*}\right)=C_{j}\left(r^{*}\right)$ and $\frac{\partial p_{B}^{*}\left(q^{*}, r^{*}\right)}{\partial q_{j}}=\frac{\partial p_{A}^{*}\left(q^{*}, r^{*}\right)}{\partial r_{j}}, j=2, \ldots, N$. Furthermore, if $C$ is separable, then $q_{j}^{*}=r_{j}^{*}$, $j=2, \ldots, N$; in other words, qualities 2 through $N$ are nondifferentiated.

Corollary 3 presents a striking result. First, the uniform distribution is the most common qualityvaluation assumption in the product-differentiation literature, and it is a step function. Under the uniform- 
distribution assumption, firms must have identical price-reaction effects. ${ }^{8}$ Next, if the cost function is separable, another common assumption in the literature, then all equilibria will have differentiation in exactly one quality!

Identical price-reaction effects and separable cost are responsible for quality nondifferentiation. Here is a simple example to show that even when there is no difference between price-reaction effects between firms, equilibrium product differentiation arises entirely because of cost consideration. Let the cost function (for a model with two qualities) be $C\left(q_{1}, q_{2}\right)=\frac{1}{2} q_{1}^{2}+\theta q_{1} q_{2}+\frac{1}{2} q_{2}^{2}$, for some parameter $\theta$. Then $C_{1}\left(q_{1}, q_{2}\right)=$ $q_{1}+\theta q_{2}$, and $C_{2}\left(q_{1}, q_{2}\right)=\theta q_{1}+q_{2}$. Suppose that $f_{1}$ is a step function, so firms' price-reaction effects are: $\frac{\partial p_{B}^{*}}{\partial q_{j}}-\frac{\partial p_{A}^{*}}{\partial r_{j}}=\frac{1}{3}\left[C_{2}\left(q_{1}, q_{2}\right)-C_{2}\left(r_{1}, r_{2}\right)\right]$. According to Corollary $3, C_{2}\left(q_{1}^{*}, q_{2}^{*}\right)=C_{2}\left(r_{1}^{*}, r_{2}^{*}\right)$. In other words, $\theta q_{1}^{*}+q_{2}^{*}=\theta r_{1}^{*}+r_{2}^{*}$, and $\theta\left(r_{1}^{*}-q_{1}^{*}\right)=q_{2}^{*}-r_{2}^{*}$. By assumption we have $q_{1}^{*}<r_{1}^{*}$ in the equilibrium. We conclude that $q_{2}^{*}>r_{2}^{*}$ if and only if $\theta>0$. When qualities have positive spillover on cost $(\theta>0)$, then Firm $A$ 's product has one superior quality and one inferior quality compared to Firm $B$ 's. By contrast, when qualities have negative spillover $(\theta<0)$, Firm $A$ 's qualities are always lower than Firm $B$ 's. (The equilibrium demand can be likened to the one in Figure 1.)

Corollary 3 has used the convention that in the equilibrium, $q_{1}^{*}<r_{1}^{*}$. The following explains the scope of the convention.

Corollary 4 Consider the game defined by valuation densities $f_{i}, i=1, \ldots, N$, and the separable cost function $C=\sum_{i=1}^{N} \gamma_{i}$. Suppose that at least one of the densities is a step function.

i) In every subgame-perfect equilibrium, each firm must choose an identical level in at least one quality.

ii) If in an equilibrium $\left(q^{*}, r^{*}\right)$ there is differentiation in the $j^{\text {th }}$ quality, so that $q_{j}^{*} \neq r_{j}^{*}$, and $f_{j}$ is a step function, $j=1, \ldots, N$, then there is no differentiation in any other quality, so $q_{k}^{*}=r_{k}^{*}$, for $k=1,2, \ldots, N$, and $k \neq j$.

In this corollary, we have gotten rid of the convention that in equilibrium Firm $A$ chooses a lower first quality than Firm $B$. Consider all equilibria of the multistage game, given valuation densities and the cost function. The effect of any uniform quality-valuation distribution and the separable cost function is

\footnotetext{
${ }^{8}$ In fact, for any subgame $(q, r)$, the difference of the price-reaction effects vanishes when $f_{1}$ is a step function.
} 
quite striking. Suppose that the $j^{\text {th }}$ quality has a uniform valuation distribution. If it so happens that in equilibrium firms choose $q_{j}^{*} \neq r_{j}^{*}$, then Corollary 3 applies to the $j^{\text {th }}$ quality, so all equilibrium qualities except the $j^{\text {th }}$ must be identical. The only case in which equilibrium differentiation happens in more than one quality is when $q_{j}^{*}=r_{j}^{*}$. Then Corollary 3 does not apply to the $j^{\text {th }}$ quality. But this means that there is (at least) one nondifferentiated quality.

Corollary 4 clarifies the "Max-Min-Min...-Min" results in Irmen and Thisse (1998). They consider an $N$ dimensional Hotelling model (which can be translated into our $N$-dimensional quality model). Consumers' locations are uniformly distributed on the $N$-dimensional unit hypercube. Consumers' mismatch disutility is the (weighted) $\mathrm{N}$-dimensional Euclidean distance. Irmen and Thisse derive a subgame-perfect equilibrium in which the two firms choose the maximum distance between themselves in one dimension but zero distance in all other dimensions (p90, Proposition 2). Although Corollary 4 does not address existence of equilibria, it is consistent with the Irmen-Thisse result. To see this, we can rewrite Corollary 4 as follows: if $M$ of the qualities have uniformly distributed valuations, $1 \leq M \leq N$, then at least $\min \{M, N-1\}$ qualities will be nondifferentiated. This is a slightly more general result than in Irmen and Thisse (1998).

In the vertical differentiation literature, Max-Min results have also figured prominently. Vandenbosch and Weinberg (1995) and Lauga and Ofek (2011) are two related papers that use a linear cost function, and restrict each of two qualities to be in its own bounded interval. In the notation here, in both papers, $N=2$, valuation density $f_{i}$ is uniform, quality $q_{i}$ is to be chosen from interval $\left[\underline{q}_{i}, \bar{q}_{i}\right], i=1,2$, and unit production cost at quality $q$ is $C(q)=c_{1} q_{1}+c_{2} q_{2}$, for constants $c_{1}$ and $c_{2}$. (In fact, the values of $c_{1}$ and $c_{2}$ are set at 0 in some parts of the analysis.) The linear cost function does not satisfy our assumption of strict convexity. Equilibrium qualities generically are corner solutions of firms' profit maximization. In other words, when the corner solution hits one quality lower bound by one firm $\left(\underline{q}_{i}\right)$, but an upper bound by the other $\left(\bar{q}_{i}\right)$, there is maximum quality differentiation. If firms' corner solutions are at the lower bound or upper bound, then there is minimum differentiation.

We can interpret Max-Min results in terms of price-reaction and Spence effects. First, according to Corollary 3 , because quality valuations are uniformly distributed, price-reaction effects are the same for both 
firms. In the race to use quality to raise the rival's price, there is no winner. Second, because the cost is linear, a quality's marginal contribution to unit production cost $C_{i}(q)$ is constant. The Spence effect generically cannot specify an interior solution for the efficient quality for the equilibrium set of indifferent consumers. The Max-Min (or Max-Max) results are driven by the combination of linear costs and uniform valuation distributions. For a model with strictly convex cost and general valuation distributions, in equilibrium each firm generically will choose a different level for each quality.

\section{Examples}

In this section, we present three sets of examples of a model with two quality dimensions $(N=2)$. The first set uses a Cobb-Douglas cost function, and various quality-valuation distributions. These examples illustrate how characterization of equilibrium prices. The second set uses a quadratic cost function and uniform-distribution valuations. We solve for the equilibrium conditions explicitly. In the third example we collapse one valuation distribution, so it becomes effectively a single dimension of quality valuations. This allows us to connect our results to earlier ones.

\subsection{Cobb-Douglas cost, and exponential, trapezoidal, and beta quality-valuation distributions}

Recall that the equilibrium set of indifferent consumers is given by $\widetilde{v}_{1}^{*}\left(v_{-1} ; q, r\right)=\alpha(q, r)-\sum_{k=2}^{N} v_{k} \beta_{k}(q, r), v_{k} \in$ $\left[\underline{v}_{k}, \bar{v}_{k}\right], k=2, \ldots, N$, where $\alpha(q, r)$ is in (10) and $\beta_{k}(q, r)$ is in (11). For the examples, we consider two qualities, so set $N=2$. We also let the valuations $v_{1}$ and $v_{2}$ be drawn from the unit interval $[0,1]$. We use a Cobb-Douglas type cost function $C(q)=q_{1}^{\gamma} q_{2}^{\delta}$, with $\gamma, \delta>1$.

Lemma 1 says we can solve for $\alpha(q, r)$ implicitly. In fact, for particular valuation distributions, we can obtain explicit solutions. This can be achieved by a program such as Mathematica, and that is what we have done. Mathematica codes are available from the authors.

The first example is the truncated exponential distribution. We set the valuation densities to $f_{i}\left(v_{i}\right)=$

$\exp \left(-\frac{v_{i}}{k_{i}}\right)\left[k_{i}\left(1-\exp \left(-\frac{1}{k_{i}}\right)\right)\right]^{-1}, i \in\{1,2\}$, where $k_{i}>0$ is the parameter for the distribution. Applying 
Lemma 1, and after integration, we obtain the implicit function that defines $\alpha(q, r)$ :

$$
\alpha=\frac{q_{1}^{\gamma} q_{2}^{\delta}-r_{1}^{\gamma} r_{2}^{\delta}}{q_{1}-r_{1}}-\frac{\left(\exp \left(\frac{1}{k_{1}}\right)+1\right)\left(\exp \left(\frac{1}{k_{2}}\right)-1\right) \exp \left(\frac{\alpha-1}{k_{1}}\right)\left(k_{1}+\frac{k_{2}\left(r_{2}-q_{2}\right)}{q_{1}-r_{1}}\right)}{\exp \left(\frac{1}{k_{2}}\right)-\exp \left(\frac{q_{2}-r_{2}}{k_{1}\left(q_{1}-r_{1}\right)}\right)}+2 k_{1} .
$$

Getting an explicit solution for $\alpha$ is infeasible, but it is unnecessary to do so.

The second example is a trapezoidal distribution. Here, we set $f_{i}\left(v_{i}\right)=1-k_{i}+2 k_{i} v_{i}, i \in\{1,2\}$, with $k_{i} \in[-1,1]$. The uniform distribution is a special case with $k_{i}=0$. Applying Lemma 1, we obtain the implicit function that defines $\alpha$ :

$$
\begin{aligned}
\alpha= & \frac{q_{1}^{\gamma} q_{2}^{\delta}-r_{1}^{\gamma} r_{2}^{\delta}}{q_{1}-r_{1}}+ \\
& \frac{6 \alpha\left[(\alpha-1) k_{2}+1\right]-\frac{\left(k_{2}+3\right)\left[(2 \alpha-1) k_{2}+1\right]\left(q_{2}-r_{2}\right)}{q_{1}-r_{1}}+\frac{k_{2}\left(k_{2}+2\right)\left(q_{2}-r_{2}\right)^{2}}{\left(q_{1}-r_{1}\right)^{2}}-3}{k_{2}\left(-6 \alpha+\frac{\left(k_{2}+3\right)\left(q_{2}-r_{2}\right)}{q_{1}-r_{1}}+3\right)-3} .
\end{aligned}
$$

This is a quadratic equation in $\alpha$, so an explicit solution for $\alpha$ is readily available (although the explicit solution consists of complicated functions of qualities).

For the special case of the uniform distribution, we have

$$
\begin{aligned}
\alpha= & \frac{q_{1}^{\gamma} q_{2}^{\delta}-r_{1}^{\gamma} r_{2}^{\delta}}{q_{1}-r_{1}}-2 \alpha+\frac{q_{2}-r_{2}}{q_{1}-r_{1}}+1 \\
& \text { or } \\
\alpha= & \frac{1}{3}\left[\frac{q_{1}^{\gamma} q_{2}^{\delta}-r_{1}^{\gamma} r_{2}^{\delta}}{q_{1}-r_{1}}+\frac{q_{2}-r_{2}}{q_{1}-r_{1}}+1\right] .
\end{aligned}
$$

Clearly, the uniform distribution makes computation easy, but it forces the firms' price-reaction effects to be identical.

Our last example is the beta distribution. We set $f_{i}\left(v_{i}\right)=\frac{v_{i}^{\left(a_{i}-1\right)}\left(1-v_{i}\right)^{\left(b_{i}-1\right)}}{\int_{0}^{1} x_{i}^{\left(a_{i}-1\right)}\left(1-x_{i}\right)^{\left(b_{i}-1\right)} d x}, i \in\{1,2\}$, with $a_{i}, b_{i}>0$. As an illustration, we let $a_{1}=a_{2}=2$ and $b_{1}=b_{2}=5$, then Lemma 1 yields the following implicit function for $\alpha$ :

$$
\begin{aligned}
\alpha= & \frac{q_{1}^{\gamma} q_{2}^{\delta}-r_{1}^{\gamma} r_{2}^{\delta}}{q_{1}-r_{1}} \\
& +\frac{1}{30} \frac{\left\{\begin{array}{c}
-4620 \alpha^{6}+792 \alpha^{5}(25 \beta+7)-2475 \alpha^{4} \beta(15 \beta+8)+1100 \alpha^{3} \beta^{2}(35 \beta+27) \\
-23100 \alpha^{2} \beta^{3}(\beta+1)+840 \alpha \beta^{4}(9 \beta+11)-42\left[(25 \beta+36) \beta^{5}+11\right]
\end{array}\right\}}{\left\{\begin{array}{c}
462 \alpha^{5}-66 \alpha^{4}(25 \beta+7)+165 \alpha^{3} \beta(15 \beta+8)- \\
55 \alpha^{2} \beta^{2}(35 \beta+27)+770 \alpha \beta^{3}(\beta+1)-14 \beta^{4}(9 \beta+11)
\end{array}\right\}},
\end{aligned}
$$


which is a sixth-order polynomial in $\alpha$.

In all the previous examples, we must have equilibrium quality differentiation in both dimensions because the distributions of the quality valuations are non-uniform and because the cost function is nonseparable. We also obtain differentiation in both dimensions when valuations are uniformly distributed and when the solution for $\alpha$ is in (38) because the cost function is nonseparable.

\subsection{Quadratic cost function and uniform distributions}

We consider again $N=2$. Now the valuations $v_{1}$ and $v_{2}$ are uniformly distributed on the interval $[1,2]$. We use the quadratic cost function

$$
C\left(q_{1}, q_{2}\right)=\frac{1}{2} q_{1}^{2}+\theta q_{1} q_{2}+\frac{1}{2} q_{2}^{2}
$$

which exhibits a positive cost spillover if and only if $\theta>0$, and which is separable if $\theta=0$. (This quadratic cost function was briefly mentioned in the discussion following Corollary 3.)

Solving for $\alpha(q, r)$ the integral equation in Lemma 1 we obtain a unique solution:

$$
\alpha(q, r)=\frac{1}{6} \frac{q_{1}^{2}+q_{2}\left(6+q_{2}\right)-r_{2}\left(6+r_{2}\right)+2 q_{1}\left(1+\theta q_{2}\right)-r_{1}\left(6+r_{1}+2 \theta r_{2}\right)}{q_{1}-r_{1}}
$$

The equilibrium set of marginal consumers is

$$
\widetilde{v}_{1}^{*}\left(v_{2} ; q, r\right)=\frac{1}{6} \frac{q_{1}^{2}+q_{2}\left(6+q_{2}\right)-r_{2}\left(6+r_{2}\right)+2 q_{1}\left(1+\theta q_{2}\right)-r_{1}\left(6+r_{1}+2 \theta r_{2}\right)}{q_{1}-r_{1}}-\frac{q_{2}-r_{2}}{q_{1}-r_{1}} v_{2}
$$

The expressions for $G\left(\alpha, \beta_{2}\right)$ and $H\left(\alpha, \beta_{2}\right)$ are

$$
\begin{aligned}
G\left(\alpha, \beta_{2}\right) & =\frac{1}{6} \frac{q_{1}^{2}+q_{2}\left(q_{2}-3\right)-r_{2}\left(r_{2}-3\right)+2 \theta q_{1} q_{2}-r_{1}\left(r_{1}+2 \theta r_{2}\right)}{q_{1}-r_{1}} \\
H\left(\alpha, \beta_{2}\right) & =1-\frac{1}{6} \frac{q_{1}^{2}+q_{2}\left(q_{2}-3\right)-r_{2}\left(r_{2}-3\right)+2 \theta q_{1} q_{2}-r_{1}\left(r_{1}+2 \theta r_{2}\right)}{q_{1}-r_{1}} .
\end{aligned}
$$

Price effects are:

$$
\begin{aligned}
& \frac{\partial p_{A}^{*}}{\partial r_{1}}=G+\left(r_{1}-q_{1}\right) \frac{\partial G}{\partial r_{1}}=\frac{1}{3}\left(r_{1}+\theta r_{2}\right) \\
& \frac{\partial p_{B}^{*}}{\partial q_{1}}=-H+\left(r_{1}-q_{1}\right) \frac{\partial H}{\partial q_{1}}=\frac{1}{3}\left(-3+q_{1}+\theta q_{2}\right) \\
& \frac{\partial p_{A}^{*}}{\partial r_{2}}=\left(r_{1}-q_{1}\right) \frac{\partial G}{\partial r_{2}}=\frac{1}{6}\left(-3+2 r_{2}+2 \theta r_{1}\right) \\
& \frac{\partial p_{B}^{*}}{\partial q_{2}}=\left(r_{1}-q_{1}\right) \frac{\partial H}{\partial q_{2}}=\frac{1}{6}\left(-3+2 q_{2}+2 \theta q_{1}\right)
\end{aligned}
$$


These price effects are simple. A firm's influence on the rival's price is independent of the rival's qualities, a consequence of the uniform-distribution assumption.

Moreover, $\frac{\partial p_{A}^{*}}{\partial r_{1}}>\frac{\partial p_{B}^{*}}{\partial q_{1}}$ for all values of $\theta$. We confirm that in any equilibrium $r_{1}>q_{1}$. However, $\frac{\partial p_{A}^{*}}{\partial r_{2}}$ can be smaller or larger than $\frac{\partial p_{B}^{*}}{\partial q_{2}}$. In fact, we also confirm Proposition 2:

$$
\frac{\partial p_{B}^{*}(q, r)}{\partial q_{2}}-\frac{\partial p_{A}^{*}(q, r)}{\partial r_{2}}=\frac{1}{3}\left[C_{2}\left(q_{1}, q_{2}\right)-C_{2}\left(r_{1}, r_{2}\right)\right]=\frac{1}{3}\left[q_{2}-r_{2}+\theta\left(q_{1}-r_{1}\right)\right]
$$

Solving the system of equations of the first-order conditions in Proposition 3 we find:

$$
q_{1}^{*}=\left(\frac{3}{4}\right) \frac{1-2 \theta}{1-\theta^{2}} ; \quad q_{2}^{*}=\left(\frac{3}{4}\right) \frac{2-\theta}{1-\theta^{2}} ; \quad r_{1}^{*}=\left(\frac{3}{4}\right) \frac{3-2 \theta}{1-\theta^{2}} ; \quad r_{2}^{*}=\left(\frac{3}{4}\right) \frac{2-3 \theta}{1-\theta^{2}}
$$

Any equilibrium qualities must be those in (42).

Moreover, substituting the previous qualities into the expressions for the price effects $\frac{\partial p_{A}^{*}(q, r)}{\partial r_{2}}$ and $\frac{\partial p_{B}^{*}(q, r)}{\partial q_{2}}$, in (40) and in (41), respectively, we verify that the price effects of the second qualities are the same at an equilibrium.

Finally, for some specific values of the parameter $\theta$, we have

$$
\begin{array}{lllll}
\text { (1) If } \theta=0, & q_{1}^{*}=3 / 4 ; & q_{2}^{*}=3 / 2 ; & r_{1}^{*}=9 / 4 ; & r_{2}^{*}=3 / 2 . \\
\text { (2) If } \theta=1 / 2, & q_{1}^{*}=0, & q_{2}^{*}=3 / 2 ; & r_{1}^{*}=2 ; & r_{2}^{*}=1 / 2 . \\
\text { (3) If } \theta=-1 / 2, & q_{1}^{*}=2 ; & q_{2}^{*}=5 / 2 ; & r_{1}^{*}=4 ; & r_{2}^{*}=7 / 2 .
\end{array}
$$

Case (1) in (43) illustrates Corollary 3: with a separable cost function and uniform distributions only the first dimension of quality is differentiated. Moreover, in equilibrium, the two firms must choose $q_{2}^{*}=$ $r_{2}^{*}=E\left[v_{2}\right]$. In Case (2), for positive cost spillover, Firm $A$ produces a superior second quality than Firm $B$. Conversely, in Case (3), for negative cost spillover, Firm $A$ produces an inferior second quality than Firm $B$.

\subsection{Collapsing one valuation distribution in a model with two qualities}

We have assumed that there are at least two qualities. However, we can easily modify the model to relate directly to Anderson, Goeree and Ramer (1997) who consider a single-dimension horizontal model with a general consumer-location distribution, and to Garella and Lambertini (2014), who consider a two-dimension vertical model with consumers having homogenous valuations on one quality. The modifications are setting $N=2$, and collapsing the valuation distribution of the second quality into a single point, say $\widehat{v}_{2}>0$. 
For given qualities $\left(q_{1}, q_{2}\right)=q$ from Firm $A$ and qualities $\left(r_{1}, r_{2}\right)=r$ from Firm $B$, if consumers value the second quality at $\widehat{v}_{2}$, the set of indifferent consumers in (3) becomes a single point. The equilibrium prices in (6) and (7) simplify to

$$
\begin{aligned}
& p_{A}^{*}-C(q)=\frac{F_{1}\left(\widetilde{v}_{1}\right)}{f_{1}\left(\widetilde{v}_{1}\right)}\left(r_{1}-q_{1}\right) \equiv G_{1}\left(\widetilde{v}_{1}\right)\left(r_{1}-q_{1}\right) \\
& p_{B}^{*}-C(r)=\frac{1-F_{1}\left(\widetilde{v}_{1}\right)}{f_{1}\left(\widetilde{v}_{1}\right)}\left(r_{1}-q_{1}\right) \equiv H_{1}\left(\widetilde{v}_{1}\right)\left(r_{1}-q_{1}\right),
\end{aligned}
$$

where

$$
\widetilde{v}_{1}\left(\widehat{v}_{2} ; p^{*}, q, r\right)=\frac{p_{B}^{*}-p_{A}^{*}}{r_{1}-q_{1}}-\widehat{v}_{2} \frac{r_{2}-q_{2}}{r_{1}-q_{1}} .
$$

Here, we have added the subscript 1 on the price-cost margins $G$ and $H$ to distinguish them from the general ones above. ${ }^{9}$ The logconcavity of $f_{1}$ implies that $G_{1}$ is strictly increasing, and that $H_{1}$ is strictly increasing (Anderson, Goeree and Ramer 1997, p106). Equations (44) and (45), together with the indifferent consumer (46), define a price equilibrium. Let $\widetilde{v}_{1}^{*}\left(\widehat{v}_{2} ; q, r\right)=\widetilde{v}_{1}\left(\widehat{v}_{2} ; p^{*}, q, r\right)$, where $p^{*}$ is the vector of equilibrium prices which are functions of $q$ and $r$. These three equations correspond to equations (2.1), (2.2) and (2.3) in Anderson, Goeree and Ramer (1997, p107).

We use (44) and (45) to substitute for equilibrium prices in (46). After some simplification we obtain the implicit definition of $\widetilde{v}_{1}^{*}$ :

$$
\widetilde{v}_{1}^{*}+G_{1}\left(\widetilde{v}_{1}^{*}\right)-H_{1}\left(\widetilde{v}_{1}^{*}\right)=\frac{\left[\widehat{v}_{2} q_{2}-C(q)\right]-\left[\widehat{v}_{2} r_{2}-C(r)\right]}{r_{1}-q_{1}} .
$$

From (47) we compute the derivatives of $\widetilde{v}_{1}^{*}$ with respect to the qualities; these correspond to the derivatives of $\alpha(q, r)$ in the proof of Lemma 3. Then we use those derivatives to simplify the price-reaction effects obtained from the explicit differentiation of (44) and (45) with respect to prices. The various steps are spelled out in the first two parts of Appendix B.

\footnotetext{
${ }^{9}$ The functions $G_{1}$ and $H_{1}$ are commonly called the reverse hazard rate and hazard rate, respectively.
} 
The first-order conditions from (34) to (37) in Proposition 3 can be simplified to:

$$
\begin{aligned}
& \frac{\partial p_{B}^{*}}{\partial q_{1}}+\widetilde{v}_{1}^{*}=C_{1}\left(q^{*}\right) \\
& \frac{\partial p_{B}^{*}}{\partial q_{2}}+\widehat{v}_{2}=C_{2}\left(q^{*}\right) \\
& \frac{\partial p_{A}^{*}}{\partial r_{1}}+\widetilde{v}_{1}^{*}=C_{1}\left(r^{*}\right) \\
& \frac{\partial p_{A}^{*}}{\partial r_{2}}+\widehat{v}_{2}=C_{2}\left(r^{*}\right) .
\end{aligned}
$$

In the third part of Appendix B, we prove that if the cost function $C(q)$ is separable, $C(q)=\gamma_{1}\left(q_{1}\right)+\gamma_{2}\left(q_{2}\right)$, then $\widehat{v}_{2}=\gamma_{2}^{\prime}\left(q_{2}^{*}\right)=\gamma_{2}^{\prime}\left(r_{2}^{*}\right)$, so $q_{2}^{*}=r_{2}^{*}$. This is a natural result: firms cannot differentiate a quality when consumers have homogenous valuations on that quality when quality costs are separable.

Finally, after putting the price-reaction effects into (48) and (50) (see the last part of Appendix B for the computation of the price-reaction effects), we show that equilibrium qualities $q_{1}^{*}$ and $r_{1}^{*}$ must satisfy:

$$
\begin{aligned}
& \widetilde{v}_{1}^{*}=C_{1}\left(q^{*}\right)+H_{1}\left(\widetilde{v}_{1}^{*}\right)-\frac{H_{1}^{\prime}\left(\widetilde{v}_{1}^{*}\right)}{1+G_{1}^{\prime}\left(\widetilde{v}_{1}^{*}\right)-H_{1}^{\prime}\left(\widetilde{v}_{1}^{*}\right)}\left[\frac{C\left(r^{*}\right)-C\left(q^{*}\right)}{r_{1}^{*}-q_{1}^{*}}-C_{1}\left(q^{*}\right)\right] \\
& \widetilde{v}_{1}^{*}=C_{1}\left(r^{*}\right)-G_{1}\left(\widetilde{v}_{1}^{*}\right)-\frac{G_{1}^{\prime}\left(\widetilde{v}_{1}^{*}\right)}{1+G_{1}^{\prime}\left(\widetilde{v}_{1}^{*}\right)-H_{1}^{\prime}\left(\widetilde{v}_{1}^{*}\right)}\left[C_{1}\left(r^{*}\right)-\frac{C\left(r^{*}\right)-C\left(q^{*}\right)}{r_{1}^{*}-q_{1}^{*}}\right],
\end{aligned}
$$

where

$$
\widetilde{v}_{1}^{*}-H_{1}\left(\widetilde{v}_{1}^{*}\right)+G_{1}\left(\widetilde{v}_{1}^{*}\right)=\frac{\left[\widehat{v}_{2} q_{2}^{*}-C\left(q^{*}\right)\right]-\left[\widehat{v}_{2} r_{2}^{*}-C\left(r^{*}\right)\right]}{r_{1}^{*}-q_{1}^{*}} .
$$

When the cost function is separable, we have $q_{2}^{*}=r_{2}^{*}$, so (54) becomes

$$
\widetilde{v}_{1}^{*}+G_{1}\left(\widetilde{v}_{1}^{*}\right)-H_{1}\left(\widetilde{v}_{1}^{*}\right)=\frac{\gamma_{1}\left(r_{1}^{*}\right)-\gamma_{1}\left(q_{1}^{*}\right)}{r_{1}^{*}-q_{1}^{*}} .
$$

Furthermore, equations (52) and (53) become

$$
\begin{aligned}
& \widetilde{v}_{1}^{*}=\gamma_{1}^{\prime}\left(q_{1}^{*}\right)+H_{1}\left(\widetilde{v}_{1}^{*}\right)-\frac{H_{1}^{\prime}\left(\widetilde{v}_{1}^{*}\right)}{1+G_{1}^{\prime}\left(\widetilde{v}_{1}^{*}\right)-H_{1}^{\prime}\left(\widetilde{v}_{1}^{*}\right)}\left[\frac{\gamma_{1}\left(r_{1}^{*}\right)-\gamma_{1}\left(q_{1}^{*}\right)}{r_{1}^{*}-q_{1}^{*}}-\gamma_{1}^{\prime}\left(q_{1}^{*}\right)\right] \\
& \widetilde{v}_{1}^{*}=\gamma_{1}^{\prime}\left(r_{1}^{*}\right)-G_{1}\left(\widetilde{v}_{1}^{*}\right)-\frac{G_{1}^{\prime}\left(\widetilde{v}_{1}^{*}\right)}{1+G_{1}^{\prime}\left(\widetilde{v}_{1}^{*}\right)-H_{1}^{\prime}\left(\widetilde{v}_{1}^{*}\right)}\left[\gamma_{1}^{\prime}\left(r_{1}^{*}\right)-\frac{\gamma_{1}\left(r_{1}^{*}\right)-\gamma_{1}\left(q_{1}^{*}\right)}{r_{1}^{*}-q_{1}^{*}}\right] .
\end{aligned}
$$

These last three equations together with the equilibrium prices correspond to equations (2.8) and (2.10) on pages 108 and 109 of Anderson, Goeree, and Ramer (1997). 
Garella and Lambertini (2014) use a discontinuous cost function: a firm producing $z$ units of the good at quality $\left(q_{1}, q_{2}\right)$ has a total cost of $c z+T\left(q_{1}, q_{2}\right)$ if $q_{1}>\underline{q}_{1}$, but only $T\left(q_{1}, q_{2}\right)$ if $q_{1}=\underline{q}_{1}$, where $\underline{q}_{1}>0$ and $c>0$ are fixed parameters, and $T$ is increasing when $q_{1}>\underline{q}_{1}$. Consumers have homogenous preferences on the second quality, but their valuations on the first quality follow a uniform distribution. They derive equilibria in which firms choose different levels in both qualities. We use a continuous cost function, but if costs are nonseparable, equilibria in our model will also exhibit differentiations in both qualities. Because of the uniform distribution, $\frac{\partial p_{B}^{*}\left(q^{*}, r^{*}\right)}{\partial q_{2}}=\frac{\partial p_{A}^{*}\left(q^{*}, r^{*}\right)}{\partial r_{2}}$, and $C_{2}\left(q^{*}\right)=C_{2}\left(r^{*}\right)$, which generally implies that $q_{2}^{*} \neq r_{2}^{*}$. The example presented just before Corollary 3 and the simulations in Subsection 4.2 can be used again for this illustration.

\section{Conclusion}

We reexamine the principle of product differentiation relaxing price competition in the classical quality-price game. The environment for analysis in our model is more general than existing works. Yet, we are able to characterize equilibria without compromise, thereby confirming the principle of differentiation. The outcome of minimum differentiation in earlier works can be attributed to consumers' quality valuations (or location) being uniformly distributed and quality cost (or mismatch disutility) being separable. The principle of product differentiation is robust: firms tend to choose different quality attributes.

Various open questions remain. First, unlike previous works that use explicit functional forms, we have used a general setup. Whereas direct computation of equilibria has been the main technique, we have used only necessary conditions of equilibria. Although we do not provide existence results, our characterization applies to each equilibrium when it does exist. The existence and uniqueness issues are beyond the scope of the current research, but it may turn out to be a rewarding endeavor. Second, our innovation is the representation of the price equilibrium by the solution of an integral equation. This technique is uncommon. In fact, economists seldom have to solve integral equations. It may well be that our technique can shed light on other models with multiple preferences or cost attributes. Third, we assume linear preferences: each quality benefits a consumer at a constant rate. The linearity assumption is so ubiquitous in modern microeconomics that relaxing this is both challenging and consequential. Finally, it seems that extending 
our environment to allow for consumers having correlated valuations between qualities may be worthwhile. Our framework may just be rich enough for this extension. One may work with conditional joint densities to derive demand functions. Of course, the exact formulation will be future research. 


\section{Appendix A: Proofs of Lemmas, Propositions, and Corollaries}

Proof of Lemma 1: Equilibrium prices $p_{A}^{*}$ and $p_{B}^{*}$ depend on qualities $(q, r)$, so the right-hand side of (8) is (affine) linear in $v_{2}, \ldots, v_{N}$. The solution $\widetilde{v}_{1}^{*}\left(v_{-1} ; q, r\right)$ of (9) also satisfies (8), so it must also be linear in $v_{2}, \ldots, v_{N}$. Therefore, we write $\widetilde{v}_{1}^{*}\left(v_{-1} ; q, r\right)=\alpha(q, r)-\sum_{k=2}^{N} v_{k} \beta_{k}(q, r), v_{k} \in\left[\underline{v}_{k}, \bar{v}_{k}\right], k=2, \ldots, N$ for some functions $\alpha$, and $\beta_{k}, k=2, \ldots, N$. Then we substitute $\widetilde{v}_{1}^{*}\left(v_{-1} ; q, r\right)$ by $\alpha(q, r)-\sum_{k=2}^{N} v_{k} \beta_{k}(q, r)$ in $(9)$ to get

$$
\begin{gathered}
\alpha(q, r)-\sum_{k=2}^{N} v_{k} \beta_{k}(q, r)=\frac{\int_{v_{-1}}\left[1-2 F_{1}\left(\alpha(q, r)-\sum_{k=2}^{N} v_{k} \beta_{k}(q, r)\right)\right] \mathrm{d} F_{-1}}{\int_{v_{-1}} f_{1}\left(\alpha(q, r)-\sum_{k=2}^{N} v_{k} \beta_{k}(q, r)\right) \mathrm{d} F_{-1}} \\
+\frac{C(r)-C(q)}{r_{1}-q_{1}}-\sum_{k=2}^{N} v_{k} \frac{r_{k}-q_{k}}{r_{1}-q_{1}}, \quad \text { for } v_{k} \in\left[\underline{v}_{k}, \bar{v}_{k}\right], k=2, \ldots, N .
\end{gathered}
$$

Because this is true for every $v_{2}, \ldots, v_{N}$, the equations (10) and (11) in the lemma follow.

Proof of Lemma 2: From the definitions (14) and (15), at each $(q, r)$, we have:

$$
G\left(\alpha, \beta_{-1}\right)+H\left(\alpha, \beta_{-1}\right)=\frac{1}{\int_{v_{-1}} f_{1}\left(\alpha-v_{-1} \cdot \beta_{-1}\right) \mathrm{d} F_{-1}}
$$

Hence

$$
\mathrm{d} \ln (G+H)+\mathrm{d} \ln \int_{v_{-1}} f_{1}\left(\alpha-v_{-1} \cdot \beta_{-1}\right) \mathrm{d} F_{-1}=0,
$$

so the first statement of the lemma follows.

Because (20) holds for each $(q, r)$, we can partially differentiate it with respect to $\alpha$ and $\beta_{j}, j=2, \ldots, N$, to obtain (21) and (22).

Proof of Lemma 3: From (11), the functions $\beta_{j}(q, r)$ are $\beta_{j}=\frac{r_{j}-q_{j}}{r_{1}-q_{1}}, \quad j=2, \ldots, N$. Hence,

$$
\frac{\partial \beta_{j}}{\partial q_{1}}=\frac{r_{j}-q_{j}}{\left(r_{1}-q_{1}\right)^{2}}=-\frac{\partial \beta_{j}}{\partial r_{1}}, \text { and } \frac{\partial \beta_{j}}{\partial q_{j}}=-\frac{1}{r_{1}-q_{1}}=-\frac{\partial \beta_{j}}{\partial r_{j}}, \quad j=2, \ldots, N,
$$

and all partial derivatives of $\beta_{j}$ with respect to $q_{k}$ or $r_{k}, k \neq j$, vanish. These prove the second equality in $(24)$.

From definitions of $G$ and $H$ in (14) and (15), we write (10) as

$$
\alpha+G\left(\alpha, \beta_{-1}\right)-H\left(\alpha, \beta_{-1}\right)=\frac{C(r)-C(q)}{r_{1}-q_{1}} .
$$


We totally differentiate (56) to obtain

$$
\left[1+\frac{\partial G}{\partial \alpha}-\frac{\partial H}{\partial \alpha}\right] \mathrm{d} \alpha+\sum_{k=2}^{N}\left(\frac{\partial G}{\partial \beta_{k}}-\frac{\partial H}{\partial \beta_{k}}\right) \mathrm{d} \beta_{j}=\mathrm{d}\left[\frac{C(r)-C(q)}{r_{1}-q_{1}}\right], \quad j=2, \ldots, N .
$$

Using (55), we obtain the partial derivatives of $\alpha$ with respect to each quality, and then simplify them to:

$$
\begin{aligned}
& {\left[1+\frac{\partial G}{\partial \alpha}-\frac{\partial H}{\partial \alpha}\right] \frac{\partial \alpha}{\partial q_{1}}=-\left[\frac{\partial G}{\partial \beta_{1}}-\frac{\partial H}{\partial \beta_{1}}\right] \frac{r_{j}-q_{j}}{\left(r_{1}-q_{1}\right)^{2}}-\frac{C_{1}(q)}{\left(r_{1}-q_{1}\right)}+\left[\frac{C(r)-C(q)}{\left(r_{1}-q_{1}\right)^{2}}\right]} \\
& {\left[1+\frac{\partial G}{\partial \alpha}-\frac{\partial H}{\partial \alpha}\right] \frac{\partial \alpha}{\partial r_{1}}=\left[\frac{\partial G}{\partial \beta_{1}}-\frac{\partial H}{\partial \beta_{1}}\right] \frac{r_{j}-q_{j}}{\left(r_{1}-q_{1}\right)^{2}}+\frac{C_{1}(r)}{\left(r_{1}-q_{1}\right)}-\left[\frac{C(r)-C(q)}{\left(r_{1}-q_{1}\right)^{2}}\right]} \\
& {\left[1+\frac{\partial G}{\partial \alpha}-\frac{\partial H}{\partial \alpha}\right] \frac{\partial \alpha}{\partial q_{j}}=\left[\frac{\partial G}{\partial \beta_{j}}-\frac{\partial H}{\partial \beta_{j}}\right] \frac{1}{\left(r_{1}-q_{1}\right)}-\frac{C_{j}(q)}{\left(r_{1}-q_{1}\right)} \quad j=2, \ldots, N} \\
& {\left[1+\frac{\partial G}{\partial \alpha}-\frac{\partial H}{\partial \alpha}\right] \frac{\partial \alpha}{\partial r_{j}}=-\left[\frac{\partial G}{\partial \beta_{j}}-\frac{\partial H}{\partial \beta_{j}}\right] \frac{1}{\left(r_{1}-q_{1}\right)}+\frac{C_{j}(r)}{\left(r_{1}-q_{1}\right)} \quad j=2, \ldots, N,}
\end{aligned}
$$

where $C_{i}(q) \equiv \frac{\partial C(q)}{\partial q_{i}}$ denotes the $i^{\text {th }}$ partial derivative of the cost function $C$. The first part of (24) follows by summing up the first two equations and the last two equations in (57) to compute $\frac{\partial \alpha(q, r)}{\partial q_{i}}+\frac{\partial \alpha(q, r)}{\partial r_{i}}$, $i=1, \ldots, N$.

Proof of Proposition 2: From (18) and (19), we have

$$
\frac{\partial p_{B}^{*}(q, r)}{\partial q_{j}}-\frac{\partial p_{A}^{*}(q, r)}{\partial r_{j}}=\left(r_{1}-q_{1}\right)\left\{\left[\frac{\partial H}{\partial \alpha} \frac{\partial \alpha}{\partial q_{j}}+\frac{\partial H}{\partial \beta_{j}} \frac{\partial \beta_{j}}{\partial q_{j}}\right]-\left[\frac{\partial G}{\partial \alpha} \frac{\partial \alpha}{\partial r_{j}}+\frac{\partial G}{\partial \beta_{j}} \frac{\partial \beta_{j}}{\partial r_{j}}\right]\right\}, \quad j=2, \ldots, N
$$

Using Lemma 3, we have $\frac{\partial \alpha}{\partial q_{j}}=\frac{C_{j}(r)-C_{j}(q)}{\left(r_{1}-q_{1}\right)\left[1+\frac{\partial G}{\partial \alpha}-\frac{\partial H}{\partial \alpha}\right]}-\frac{\partial \alpha}{\partial r_{j}}$ and $\frac{\partial \beta_{j}}{\partial q_{j}}=-\frac{\partial \beta_{j}}{\partial r_{j}}$, and substitute them into the above to obtain:

$$
\begin{aligned}
& \frac{\partial p_{B}^{*}(q, r)}{\partial q_{j}}-\frac{\partial p_{A}^{*}(q, r)}{\partial r_{j}} \\
= & \left(r_{1}-q_{1}\right)\left\{\left[\frac{\partial H}{\partial \alpha}\left(\frac{C_{j}(r)-C_{j}(q)}{\left(r_{1}-q_{1}\right)\left[1+\frac{\partial G}{\partial \alpha}-\frac{\partial H}{\partial \alpha}\right]}-\frac{\partial \alpha}{\partial r_{j}}\right)-\frac{\partial H}{\partial \beta_{j}} \frac{\partial \beta_{j}}{\partial r_{j}}\right]-\left[\frac{\partial G}{\partial \alpha} \frac{\partial \alpha}{\partial r_{j}}+\frac{\partial G}{\partial \beta_{j}} \frac{\partial \beta_{j}}{\partial r_{j}}\right]\right\} \\
= & \left(r_{1}-q_{1}\right)\left\{\left[-\frac{\partial H}{\partial \alpha} \frac{\partial \alpha}{\partial r_{j}}-\frac{\partial H}{\partial \beta_{j}} \frac{\partial \beta_{j}}{\partial r_{j}}\right]-\left[\frac{\partial G}{\partial \alpha} \frac{\partial \alpha}{\partial r_{j}}+\frac{\partial G}{\partial \beta_{j}} \frac{\partial \beta_{j}}{\partial r_{j}}\right]\right\}+\frac{\partial H}{\partial \alpha} \frac{\left[C_{j}(r)-C_{j}(q)\right]}{\left[1+\frac{\partial G}{\partial \alpha}-\frac{\partial H}{\partial \alpha}\right]} \\
= & -\left(r_{1}-q_{1}\right)\left\{\left[\frac{\partial G}{\partial \alpha}+\frac{\partial H}{\partial \alpha}\right] \frac{\partial \alpha}{\partial r_{j}}+\left[\frac{\partial G}{\partial \beta_{j}}+\frac{\partial H}{\partial \beta_{j}}\right] \frac{\partial \beta_{j}}{\partial r_{j}}\right\}+\frac{\partial H}{\partial \alpha} \frac{\left[C_{j}(r)-C_{j}(q)\right]}{\left[1+\frac{\partial G}{\partial \alpha}-\frac{\partial H}{\partial \alpha}\right]} .
\end{aligned}
$$


Next, we define

$$
Z \equiv \frac{\partial H}{\partial \alpha} \frac{1}{\left[1+\frac{\partial G}{\partial \alpha}-\frac{\partial H}{\partial \alpha}\right]}
$$

We use (21) and (22) in Lemma 2 to obtain

$$
\begin{aligned}
& \frac{\partial p_{B}^{*}(q, r)}{\partial q_{j}}-\frac{\partial p_{A}^{*}(q, r)}{\partial r_{j}} \\
= & \frac{\left(r_{1}-q_{1}\right) \int_{v_{-1}} f_{1}^{\prime}\left(\alpha-v_{-1} \cdot \beta_{-1}\right) \mathrm{d} F_{-1}}{\left(\int_{v_{-1}} f_{1}\left(\alpha-v_{-1} \cdot \beta_{-1}\right) \mathrm{d} F_{-1}\right)^{2}} \frac{\partial \alpha}{\partial r_{j}}-\frac{\left(r_{1}-q_{1}\right) \int_{v_{-1}} f_{1}^{\prime}\left(\alpha-v_{-1} \cdot \beta_{-1}\right) v_{j} \mathrm{~d} F_{-1}}{\left(\int_{v_{-1}} f_{1}\left(\alpha-v_{-1} \cdot \beta_{-1}\right) \mathrm{d} F_{-1}\right)^{2}} \frac{\partial \beta_{j}}{\partial r_{j}}+Z\left[C_{j}(q)-C_{j}(r)\right] \\
= & \frac{\left(r_{1}-q_{1}\right)\left(\int_{v_{-1}} f_{1}^{\prime}\left(\alpha-v_{-1} \cdot \beta_{-1}\right) \mathrm{d} F_{-1} \frac{\partial \alpha}{\partial r_{j}}-\int_{v_{-1}} f_{1}^{\prime}\left(\alpha-v_{-1} \cdot \beta_{-1}\right) v_{j} \mathrm{~d} F_{-1} \frac{\partial \beta_{j}}{\partial r_{j}}\right)}{\left(\int_{v_{-1}} f_{1}\left(\alpha-v_{-1} \cdot \beta_{-1}\right) \mathrm{d} F_{-1}\right)^{2}}+Z\left[C_{j}(q)-C_{j}(r)\right],(58)
\end{aligned}
$$

which gives (25). Then we further write the first term in (58) as

$$
\frac{\left(r_{1}-q_{1}\right) \frac{\partial}{\partial r_{j}}\left[\int_{v_{-1}} f_{1}\left(\alpha-v_{-1} \cdot \beta_{-1}\right) \mathrm{d} F_{-1}\right]}{\left(\int_{v_{-1}} f_{1}\left(\alpha-v_{-1} \cdot \beta_{-1}\right) \mathrm{d} F_{-1}\right)^{2}},
$$

which gives the first term in (26). Finally, from Lemma 3, we have $\frac{\partial \alpha}{\partial r_{j}}=\frac{C_{j}(r)-C_{j}(q)}{\left(r_{1}-q_{1}\right)\left[1+\frac{\partial G}{\partial \alpha}-\frac{\partial H}{\partial \alpha}\right]}-\frac{\partial \alpha}{\partial q_{j}}$ and $\frac{\partial \beta_{j}}{\partial r_{j}}=-\frac{\partial \beta_{j}}{\partial q_{j}}$, so (59) also equals

$$
-\frac{\left(r_{1}-q_{1}\right) \frac{\partial}{\partial q_{j}}\left[\int_{v_{-1}} f_{1}\left(\alpha-v_{-1} \cdot \beta_{-1}\right) \mathrm{d} F_{-1}\right]}{\left(\int_{v_{-1}} f_{1}\left(\alpha-v_{-1} \cdot \beta_{-1}\right) \mathrm{d} F_{-1}\right)^{2}},
$$

which gives the first term in (27).

Finally, from the definition of $G\left(\alpha, \beta_{-1}\right)$ and $H\left(\alpha, \beta_{-1}\right)$ in (14) and (15), we have:

$$
\begin{aligned}
& \frac{\partial}{\partial \alpha} G\left(\alpha, \beta_{-1}\right)= 1-\frac{\int_{v_{-1}} f_{1}^{\prime}\left(\alpha-v_{-1} \cdot \beta_{-1}\right) \mathrm{d} F_{-1} \int_{v_{-1}} F_{1}\left(\alpha-v_{-1} \cdot \beta_{-1}\right) \mathrm{d} F_{-1}}{\left(\int_{v_{-1}} f_{1}\left(\alpha-v_{-1} \cdot \beta_{-1}\right) \mathrm{d} F_{-1}\right)^{2}} \\
& \frac{\partial}{\partial \alpha} H\left(\alpha, \beta_{-1}\right)=-1-\frac{\int_{v_{-1}} f_{1}^{\prime}\left(\alpha-v_{-1} \cdot \beta_{-1}\right) \mathrm{d} F_{-1} \int_{v_{-1}}\left[1-F_{1}\left(\alpha-v_{-1} \cdot \beta_{-1}\right)\right] \mathrm{d} F_{-1}}{\left(\int_{v_{-1}} f_{1}\left(\alpha-v_{-1} \cdot \beta_{-1}\right) \mathrm{d} F_{-1}\right)^{2}} .
\end{aligned}
$$


After we substitute these into the definition of $Z$, we obtain the same expression for $Z$ in the Proposition.

Proof of Proposition 3: We begin by simplifying Firm A's first-order derivatives with respect to qualities. First, for (30) we use (3) to obtain

$$
\begin{aligned}
& \frac{\partial}{\partial q_{j}} \int_{v_{-1}} F_{1}\left(\widetilde{v}_{1}\left(v_{-1} ; p^{*}\left(q, r^{*}\right), q, r^{*}\right)\right) \mathrm{d} F_{-1} \\
= & \frac{1}{r_{1}-q_{1}} \int_{v_{-1}} f_{1}\left(\widetilde{v}_{1}\left(v_{-1} ; p^{*}\left(q, r^{*}\right), q, r^{*}\right)\right) v_{j} \mathrm{~d} F_{-1} \quad j=2, \ldots, N .
\end{aligned}
$$

Second, for (31), again we use (3) to obtain

$$
\begin{aligned}
& \frac{\partial}{\partial p_{B}^{*}} \int_{v_{-1}} F_{1}\left(\widetilde{v}_{1}\left(v_{-1} ; p^{*}\left(q, r^{*}\right), q, r^{*}\right)\right) \mathrm{d} F_{-1} \\
= & \frac{1}{r_{1}-q_{1}} \int_{v_{-1}} f_{1}\left(\widetilde{v}_{1}\left(v_{-1} ; p^{*}\left(q, r^{*}\right), q, r^{*}\right)\right) \mathrm{d} F_{-1} .
\end{aligned}
$$

We then substitute these expressions into (30) and (31), and the first-order derivative of Firm $A$ 's with respect to quality $q_{j}, j=2, \ldots, N$, becomes

$$
\begin{aligned}
& -\left[\int_{v_{-1}} F_{1}\left(\widetilde{v}_{1}\left(v_{-1} ; p^{*}\left(q, r^{*}\right), q, r^{*}\right)\right) \mathrm{d} F_{-1}\right] C_{i}(q) \\
& +\frac{1}{r_{1}-q_{1}} \int_{v_{-1}} f_{1}\left(\widetilde{v}_{1}\left(v_{-1} ; p^{*}\left(q, r^{*}\right), q, r^{*}\right)\right) v_{j} \mathrm{~d} F_{-1}\left[p_{A}^{*}\left(q, r^{*}\right)-C(q)\right] \\
& +\frac{1}{r_{1}-q_{1}} \int_{v_{-1}} f_{1}\left(\widetilde{v}_{1}\left(v_{-1} ; p^{*}\left(q, r^{*}\right), q, r^{*}\right)\right) \mathrm{d} F_{-1} \frac{\partial p_{B}^{*}}{\partial q_{i}}\left[p_{A}^{*}\left(q, r^{*}\right)-C(q)\right] .
\end{aligned}
$$

We now evaluate (60) at the equilibrium qualities, so replace $\left.\widetilde{v}_{1}\left(v_{-1} ; p^{*}\left(q^{*}, r^{*}\right), q^{*}, r^{*}\right)\right)$ as $\widetilde{v}_{1}^{*}\left(v_{-1} ; q^{*}, r^{*}\right)=$ $\alpha\left(q^{*}, r^{*}\right)-v_{-1} \cdot \beta_{-1}\left(q^{*}, r^{*}\right)$. Using the equilibrium price (12) in Proposition 1

$$
\frac{p_{A}^{*}\left(q^{*}, r^{*}\right)-C\left(q^{*}\right)}{r_{1}^{*}-q_{1}^{*}}=\frac{\left.\int_{v_{-1}} F_{1}\left(\alpha-v_{-1} \cdot \beta_{-1}\right)\right) \mathrm{d} F_{-1}}{\left.\int_{v_{-1}} f_{1}\left(\alpha-v_{-1} \cdot \beta_{-1}\right)\right) \mathrm{d} F_{-1}}
$$

we simplify the first-order derivative of Firm $A$ 's profit with respect to $q_{j}$ to

$$
\left[\int_{v_{-1}} F_{1}\left(\alpha-v_{-1} \cdot \beta_{-1}\right) \mathrm{d} F_{-1}\right]\left[\frac{\partial p_{B}^{*}}{\partial q_{j}}+\frac{\int_{v_{-1}} f_{1}\left(\alpha-v_{-1} \cdot \beta_{-1}\right) v_{j} \mathrm{~d} F_{-1}}{\left.\int_{v_{-1}} f_{1}\left(\alpha-v_{-1} \cdot \beta_{-1}\right)\right) \mathrm{d} F_{-1}}-C_{j}(q)\right], \quad j=2, \ldots, N,
$$

where we have omitted the arguments in $\alpha$ and $\beta_{-1}$. We set this to zero to obtain the first-order condition for $q_{j}^{*}$ :

$$
\frac{\partial p_{B}^{*}}{\partial q_{j}}+\frac{\int_{v_{-1}} f_{1}\left(\alpha-v_{-1} \cdot \beta_{-1}\right) v_{j} \mathrm{~d} F_{-1}}{\left.\int_{v_{-1}} f_{1}\left(\alpha-v_{-1} \cdot \beta_{-1}\right)\right) \mathrm{d} F_{-1}}-C_{j}\left(q^{*}\right)=0 \quad j=2, \ldots, N .
$$


For brevity we do not lay out all the steps for obtaining the first-order condition of Firm $A$ 's equilibrium quality $q_{1}$, but the key difference is that (3) yields $\frac{\partial \widetilde{v}_{1}}{\partial q_{1}}=\frac{\widetilde{v}_{1}}{r_{1}-q_{1}}$. The effect of quality $q_{1}$ on demand now becomes

$$
\begin{aligned}
& \frac{\partial}{\partial q_{1}} \int_{v_{-1}} F_{1}\left(\widetilde{v}_{1}\left(v_{-1} ; p^{*}\left(q, r^{*}\right), q, r^{*}\right)\right) \mathrm{d} F_{-1} \\
= & \frac{1}{r_{1}-q_{1}} \int_{v_{-1}} f_{1}\left(\widetilde{v}_{1}\left(v_{-1} ; p^{*}\left(q, r^{*}\right), q, r^{*}\right)\right) \tilde{v}_{1} \mathrm{~d} F_{-1} .
\end{aligned}
$$

Following the same steps, we obtain the following first-order condition for $q_{1}^{*}$

$$
\frac{\partial p_{B}^{*}}{\partial q_{1}}+\frac{\int_{v_{-1}} f_{1}\left(\alpha-v_{-1} \cdot \beta_{-1}\right) \widetilde{v}_{1}^{*} \mathrm{~d} F_{-1}}{\left.\int_{v_{-1}} f_{1}\left(\alpha-v_{-1} \cdot \beta_{-1}\right)\right) \mathrm{d} F_{-1}}-C_{1}\left(q^{*}\right)=0 .
$$

The first-order conditions for Firm B's equilibrium qualities are derived analogously, so we do not repeat the steps here.

Proof of Corollary 1: For each $j=2, \ldots, N$, the terms with the integrals are the same in the two equations in (36) and (37). Taking their difference, we have

$$
\frac{\partial p_{B}^{*}}{\partial q_{j}}-\frac{\partial p_{A}^{*}}{\partial r_{j}}=C_{j}\left(q^{*}\right)-C_{j}\left(r^{*}\right)
$$

and the equivalence of i) and ii) in the Corollary follows. Then we simply apply Proposition 2 on the equilibrium $\left(q^{*}, r^{*}\right)$ for the equivalence of ii) and iii).

Proof of Corollary 3: We actually prove a more general result for the first part. Consider any subgame defined by equilibrium quality vectors $(q, r)$. The difference in the firms' price-reaction effects is in Proposition 2. Obviously, $f_{1}^{\prime}=0$ by assumption, so (25) becomes $\frac{\partial p_{B}^{*}(q, r)}{\partial q_{j}}-\frac{\partial p_{A}^{*}(q, r)}{\partial r_{j}}=\frac{1}{3}\left[C_{j}(q)-C_{j}(r)\right]$. Under the step-function assumption, Corollary 1 then says that $\frac{\partial p_{B}^{*}\left(q^{*}, r^{*}\right)}{\partial q_{j}}-\frac{\partial p_{A}^{*}\left(q^{*}, r^{*}\right)}{\partial r_{j}}=\frac{1}{3}\left[C_{j}\left(q^{*}\right)-C_{j}\left(r^{*}\right)\right]=$ $C_{j}\left(q^{*}\right)-C_{j}\left(r^{*}\right)$. Hence it must be $C_{j}\left(q^{*}\right)=C_{j}\left(r^{*}\right), j=2, \ldots, N$. Which also implies that, in equilibrium, $\frac{\partial p_{B}^{*}\left(q^{*}, r^{*}\right)}{\partial q_{j}}-\frac{\partial p_{A}^{*}\left(q^{*}, r^{*}\right)}{\partial r_{j}}=0$. Finally, we apply Corollary 2 to obtain the nondifferentiation result.

Proof of Corollary 4: Let the valuation density of quality $j$ be uniform. Consider an equilibrium $\left(q^{*} r^{*}\right)$. If $q_{j}^{*}=r_{j}^{*}$, then the first part of the corollary is trivially true. Suppose that $q_{j}^{*} \neq r_{j}^{*}$. Without loss of generality we let $q_{j}^{*}<r_{j}^{*}$. Now we relabel the indexes so that $j=1$. Then Corollary 3 applies, and the firms choose identical qualities for all quality attributes $k=2, \ldots, N$. 


\section{Appendix B: On collapsing one valuation distribution in a model with two qualities}

\section{Derivatives of $\widetilde{v}_{1}^{*}$ with respect to qualities}

From (47) we obtain the derivatives of $\widetilde{v}_{1}^{*}$ in (54) with respect to the four qualities:

$$
\begin{aligned}
{\left[1+G_{1}^{\prime}\left(\widetilde{v}_{1}^{*}\right)-H_{1}^{\prime}\left(\widetilde{v}_{1}^{*}\right)\right] \frac{\partial \widetilde{v}_{1}^{*}}{\partial q_{1}} } & =\frac{\left[\widehat{v}_{2} q_{2}-C(q)\right]-\left[\widehat{v}_{2} r_{2}-C(r)\right]}{\left(r_{1}-q_{1}\right)^{2}}-\frac{C_{1}(q)}{r_{1}-q_{1}} \\
{\left[1+G_{1}^{\prime}\left(\widetilde{v}_{1}^{*}\right)-H_{1}^{\prime}\left(\widetilde{v}_{1}^{*}\right)\right] \frac{\partial \widetilde{v}_{1}^{*}}{\partial q_{2}} } & =\frac{\widehat{v}_{2}-C_{2}(q)}{r_{1}-q_{1}} \\
{\left[1+G_{1}^{\prime}\left(\widetilde{v}_{1}^{*}\right)-H_{1}^{\prime}\left(\widetilde{v}_{1}^{*}\right)\right] \frac{\partial \widetilde{v}_{1}^{*}}{\partial r_{1}} } & =-\frac{\left[\widehat{v}_{2} q_{2}-C(q)\right]-\left[\widehat{v}_{2} r_{2}-C(r)\right]}{\left(r_{1}-q_{1}\right)^{2}}+\frac{C_{1}(r)}{r_{1}-q_{1}} \\
{\left[1+G_{1}^{\prime}\left(\widetilde{v}_{1}^{*}\right)-H_{1}^{\prime}\left(\widetilde{v}_{1}^{*}\right)\right] \frac{\partial \widetilde{v}_{1}^{*}}{\partial r_{2}} } & =-\frac{\widehat{v}_{2}-C_{2}(r)}{r_{1}-q_{1}} .
\end{aligned}
$$

These are the corresponding derivatives of $\alpha(q, r)$ in the proof of Lemma 3.

\section{Price-reaction effects}

Next, from (44) and (45) we obtain the following:

$$
\begin{aligned}
\frac{\partial p_{A}^{*}}{\partial r_{1}} & =G_{1}\left(\widetilde{v}_{1}^{*}\right)+\left(r_{1}-q_{1}\right) G_{1}^{\prime}\left(\widetilde{v}_{1}^{*}\right) \frac{\partial \widetilde{v}_{1}^{*}}{\partial r_{1}} \\
\frac{\partial p_{A}^{*}}{\partial r_{2}} & =\left(r_{1}-q_{1}\right) G_{1}^{\prime}\left(\widetilde{v}_{1}^{*}\right) \frac{\partial \widetilde{v}_{1}^{*}}{\partial r_{2}} \\
\frac{\partial p_{B}^{*}}{\partial q_{1}} & =-H_{1}\left(\widetilde{v}_{1}^{*}\right)+\left(r_{1}-q_{1}\right) H_{1}^{\prime}\left(\widetilde{v}_{1}^{*}\right) \frac{\partial \widetilde{v}_{1}^{*}}{\partial q_{1}} \\
\frac{\partial p_{B}^{*}}{\partial q_{2}} & =\left(r_{1}-q_{1}\right) H_{1}^{\prime}\left(\widetilde{v}_{1}^{*}\right) \frac{\partial \widetilde{v}_{1}^{*}}{\partial q_{2}} .
\end{aligned}
$$

The four price-reaction effects can be obtained after the derivatives of $\widetilde{v}_{1}^{*}$ with respect to qualities are substituted by those above.

Firms choosing the same second quality under separable cost function

We now claim that $q_{2}^{*}=r_{2}^{*}$ so the two firms choose the same level for the second quality when cost is 
separable. To see this, use the expression $\frac{\partial \widetilde{v}_{1}^{*}}{\partial r_{2}}$ for $(61)$ to obtain

$$
\frac{\partial p_{A}^{*}}{\partial r_{2}}=-\left[\frac{G_{1}^{\prime}\left(\widetilde{v}_{1}^{*}\right)}{1+G_{1}^{\prime}\left(\widetilde{v}_{1}^{*}\right)-H_{1}^{\prime}\left(\widetilde{v}_{1}^{*}\right)}\right]\left[\widehat{v}_{2}-\gamma_{2}^{\prime}\left(r_{2}^{*}\right)\right],
$$

and use the expression $\frac{\partial \widetilde{v}_{1}^{*}}{\partial q_{2}}$ above for (62) to obtain

$$
\frac{\partial p_{B}^{*}}{\partial q_{2}}=\left[\frac{H_{1}^{\prime}\left(\widetilde{v}_{1}^{*}\right)}{1+G_{1}^{\prime}\left(\widetilde{v}_{1}^{*}\right)-H_{1}^{\prime}\left(\widetilde{v}_{1}^{*}\right)}\right]\left[\widehat{v}_{2}-\gamma_{2}^{\prime}\left(q_{2}^{*}\right)\right] .
$$

Then we substitute them into (49) and (51) to obtain

$$
\begin{array}{r}
{\left[\frac{H_{1}^{\prime}\left(\widetilde{v}_{1}^{*}\right)}{1+G_{1}^{\prime}\left(\widetilde{v}_{1}^{*}\right)-H_{1}^{\prime}\left(\widetilde{v}_{1}^{*}\right)}\right]\left[\widehat{v}_{2}-\gamma_{2}^{\prime}\left(q_{2}\right)\right]+\widehat{v}_{2}=\gamma_{2}^{\prime}\left(q_{2}^{*}\right)} \\
-\left[\frac{G_{1}^{\prime}\left(\widetilde{v}_{1}^{*}\right)}{1+G_{1}^{\prime}\left(\widetilde{v}_{1}^{*}\right)-H_{1}^{\prime}\left(\widetilde{v}_{1}^{*}\right)}\right]\left[\widehat{v}_{2}-\gamma_{2}^{\prime}\left(r_{2}^{*}\right)\right]+\widehat{v}_{2}=\gamma_{2}^{\prime}\left(r_{2}^{*}\right)
\end{array}
$$

which simplify to

$$
\begin{aligned}
& {\left[1+\frac{H_{1}^{\prime}\left(\widetilde{v}_{1}^{*}\right)}{1+G_{1}^{\prime}\left(\widetilde{v}_{1}^{*}\right)-H_{1}^{\prime}\left(\widetilde{v}_{1}^{*}\right)}\right]\left[\widehat{v}_{2}-\gamma_{2}^{\prime}\left(q_{2}\right)\right]=0} \\
& {\left[1-\frac{G_{1}^{\prime}\left(\widetilde{v}_{1}^{*}\right)}{1+G_{1}^{\prime}\left(\widetilde{v}_{1}^{*}\right)-H_{1}^{\prime}\left(\widetilde{v}_{1}^{*}\right)}\right]\left[\widehat{v}_{2}-\gamma_{2}^{\prime}\left(r_{2}^{*}\right)\right]=0,}
\end{aligned}
$$

so we have $q_{2}^{*}=r_{2}^{*}$.

Price-reaction effects for characterizing equilibrium qualities $q_{1}^{*}$ and $r_{1}^{*}$

We use $\frac{\partial \widetilde{v}_{1}^{*}}{\partial q_{1}}$ to compute

$$
\begin{aligned}
\frac{\partial p_{B}^{*}}{\partial q_{1}} & =-H_{1}\left(\widetilde{v}_{1}^{*}\right)+\left(r_{1}-q_{1}\right) H_{1}^{\prime}\left(\widetilde{v}_{1}^{*}\right) \frac{\partial \widetilde{v}_{1}^{*}}{\partial q_{1}} \\
& =-H_{1}\left(\widetilde{v}_{1}^{*}\right)+\frac{H_{1}^{\prime}\left(\widetilde{v}_{1}^{*}\right)}{1+G_{1}^{\prime}\left(\widetilde{v}_{1}^{*}\right)-H_{1}^{\prime}\left(\widetilde{v}_{1}^{*}\right)}\left[\frac{C\left(r^{*}\right)-C\left(q^{*}\right)}{r_{1}^{*}-q_{1}^{*}}-C_{1}\left(q^{*}\right)\right]<0
\end{aligned}
$$

which exhibits the classical price reaction. Because $q_{1}<r_{1}$, if Firm $A$ raises $q_{1}$, the difference between the two qualities drops, and Firm $B$ reacts by reducing its price. Similarly, we use $\frac{\partial \widetilde{v}_{1}}{\partial r_{1}}$ to compute

$$
\begin{aligned}
\frac{\partial p_{A}^{*}}{\partial r_{1}} & =G_{1}\left(\widetilde{v}_{1}^{*}\right)+\left(r_{1}^{*}-q_{1}^{*}\right) G_{1}^{\prime}\left(\widetilde{v}_{1}^{*}\right) \frac{\partial \widetilde{v}_{1}}{\partial r_{1}} \\
& =G_{1}\left(\widetilde{v}_{1}^{*}\right)+\frac{G_{1}^{\prime}\left(\widetilde{v}_{1}^{*}\right)}{1+G_{1}^{\prime}\left(\widetilde{v}_{1}^{*}\right)-H_{1}^{\prime}\left(\widetilde{v}_{1}^{*}\right)}\left[C_{1}\left(r^{*}\right)-\frac{C\left(r^{*}\right)-C\left(q^{*}\right)}{r_{1}^{*}-q_{1}^{*}}\right]>0,
\end{aligned}
$$

which again has the classical price reaction. 


\section{References}

[1] Anderson, S., A. De Palma, and J.-F. Thisse (1992). Discrete choice theory of product differentiation, MIT press, Cambridge, MA.

[2] Anderson, S., J. Goeree, and R. Ramer (1997). "Location, location, location," Journal of Economic Theory 77(1):102-127.

[3] Ansari A., N. Economides, and J. Steckel (1998). "The max-min-min principle of product differentiation," Journal of Regional Science 38(2):207-230.

[4] Belleflamme, P., and M. Peitz (2010). Industrial Organization: Markets and Strategies, Cambridge University Press, New York.

[5] Benassi, C., A. Chirco, and C. Colombo (2006) "Vertical differentiation and the distribution of income," Bulletin of Economic Research 58(4):345-367.

[6] Cabral, L. (2000). Introduction to industrial organization, MIT press, Cambridge, MA.

[7] Caplin, A., and B. Nalebuff (1991). "Aggregation and imperfect competition: On the existence of equilibrium," Econometrica 59(1):25-59.

[8] Carlton, D., and J. Perloff (2005). Modern industrial organization 4th Ed, Pearson/Addison Wesley, Reading, MA.

[9] Champsaur, P., and J.-C. Rochet (1989). "Multiproduct duopolists," Econometrica 57(3):533-557.

[10] Cremer, H. and J.-F. Thisse (1991). "Location models of horizontal differentiation: a special case of vertical differentiation," Journal of Industrial Economics 39(4):383 390.

[11] D'Aspremont, C., J. Gabszewicz, and J.-F. Thisse (1979). "Hotellings stability in competition," Econometrica 47(5):1145-1150.

[12] Degryse, H. and A. Irmen (2001). "Attribute dependence and the provision of quality," Regional Science and Urban Economics 31(5):547-569. 
[13] Gabszewicz, J., and J.-F. Thisse (1979). "Price competition, quality and income disparities," Journal of Economic Theory 20:340-359.

[14] Garella, P.G., and L. Lambertini (2014). "Bidimensional vertical differentiation," International Journal of Industrial Organization 32:1-10.

[15] Hotelling, H., (1929). "Stability in competition," Economic Journal 39:41-57.

[16] Irmen, A., and J.-F.Thisse (1998). "Competition in multi-characteristics space: Hotelling was almost right," Journal of Economic Theory 78:76-102.

[17] Lauga, D., and E. Ofek (2011). "Product positioning in a two-dimensional vertical differentiation model: the role of quality costs," Marketing Science 30(5):903-923.

[18] Loertscher, S., and G. Muehlheusser (2011). "Sequential location games," RAND Journal of Economics $42(4): 639-663$

[19] Ma, C., and J. Burgess (1993). "Quality competition, welfare, and regulation," Journal of Economics $58(2): 153-173$

[20] Neven, D. (1986), "On Hotelling's competition with non-uniform consumer distributions," Economics Letters 21:121-126.

[21] Pepall, L., D. Richards, and G. Norman (2014). Industrial organization: contemporary theory and empirical applications 5th Ed, Wiley, Malden, MA.

[22] Shaked, A., and J. Sutton (1982). "Relaxing price competition through product differentiation," Review of Economic Studies 49(1):3-13.

[23] Shaked, A., and J. Sutton (1983). "Natural oligopolies," Econometrica 51(5):1469-1483.

[24] Spence A.M. (1975) "Monopoly, quality and regulation," Bell Journal of Economics 6(2):417-429.

[25] Tabuchi, T. (1994). "Two-stage two dimensional spatial competition between two firms," Regional Science and Urban Economics 24:207-227. 
[26] Tabuchi, T., and J.-F. Thisse (1995). "Asymmetric equilibria in spatial competition," International Journal of Industrial Organization 13:213-227.

[27] Tirole, J. (1988). The theory of industrial organization, MIT press, Cambridge, MA.

[28] Vandenbosch, M., and C. Weinberg (1995). "Product and price competition in a two dimensional vertical differentiation model," Marketing Science 14(2):224-249.

[29] Vendorp, E.C.H., and A. Majeed (1995). "Differentiation in a two dimensional market," Regional Science and Urban Economics 25:75-83.

[30] Yurko, A. (2010). "How does income inequality affect market outcomes in vertically differentiated markets?" International Journal of Industrial Organization 29:493-503. 


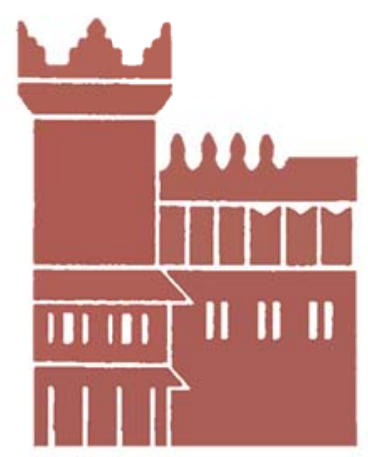

Alma Mater Studiorum - Università di Bologna DEPARTMENT OF ECONOMICS

Strada Maggiore 45

40125 Bologna - Italy

Tel. +39051 2092604

Fax +390512092664

http://www.dse.unibo.it 